\title{
COMPACT CONSTANT MEAN CURVATURE SURFACES WITH LOW GENUS
}

\author{
KARSTEN GROSSE-BRAUCKMANN AND KONRAD POLTHIER
}

\begin{abstract}
We describe numerical experiments that suggest the existence of compact constant mean curvature surfaces. Our surfaces come in three dihedrally symmetric families with the genus ranging from 3 to 5,7 to 10, and 3 to 9 , respectively; there are further surfaces with the symmetry of the Platonic polyhedra and genera 6,12 , and 30 . We use the algorithm of Oberknapp and the second author that defines a discrete version of Lawson's conjugate surface method.
\end{abstract}

Surfaces with constant mean curvature $H$ have been studied for a long time. Until recently most known boundaryless or complete surfaces were minimal $(H=0)$; the only surfaces with non-zero constant $H$ were Delaunay's surfaces of revolution [Delaunay 1841]. Compact surfaces have attracted particular attention. Whereas the maximum principle rules out the existence of compact minimal surfaces, some constructions for $H \neq 0$ are known by now. As we describe below, the resulting compact surfaces are rather complicated and also not quite as explicit as one might hope. In the present work we compute surfaces which are geometrically simpler: they are small and have a large symmetry group.

Let us assume $H$ is a nonzero constant and normalize it to 1 by a scaling; we use the shorthand notation CMC for this case. The simplest compact CMC surface is the unit sphere. The sphere is known as the unique embedded CMC surface [Alexandrov 1958], and also the unique immersed CMC sphere [Hopf 1956]. Both results focused much attention to the existence problem for further compact CMC surfaces. It is also interesting that the sphere is the only complete CMC surface which is a minimum for the variational problem for constant mean curvature [Barbosa and do Carmo 1976]: find critical levels of area for a given enclosed volume. The partial differential equation $H=$ const. can be considered the Euler equation to the variational problem.

Wente's existence proof for CMC tori [Wente 1986] was a surprising event which triggered further discoveries. Pinkall and Sterling [Pinkall and Sterling 1989] characterize all immersions that cover CMC tori, and Bobenko [Bobenko 1991] gives explicit formulae for their induced metrics in terms of theta functions. These immersions lead to compact CMC tori only if all periods vanish, otherwise to non-compact periodic surfaces. It is known that the period condition is in fact satisfied in some

Date: Sept. 95, revised Sept. 96. 
cases [Ercolani et al. 1993]. In his numerical work Heil [Heil 1995] evaluates the theta functions and studies the period problem.

Kapouleas [Kapouleas 1991] constructs a large class of compact CMC surfaces for every genus $g \geq 3$. Kapouleas glues pieces of Delaunay surfaces onto spheres and proves existence of nearby smooth CMC surfaces by an implicit function theorem argument. The Delaunay pieces must be long, and all necksizes tiny - how long and how thin precisely is the result of delicate estimates and hence practically not accessible. In [Kapouleas 1995] surfaces of every genus $g \geq 2$ are constructed using $g$ Wente tori that are glued together at a single lobe. Similar to the case of Kapouleas' Delaunay-like surfaces the fused Wente tori are almost degenerate: they have a large number of almost spherical lobes joined by necks of large Gauß curvature.

We use the algorithm of Oberknapp and Polthier [Oberknapp and Polthier] to construct compact surfaces numerically. This algorithm gives a discrete version of the conjugate surface construction which was introduced by Lawson [Lawson 1970]. Lawson constructed two doubly periodic CMC surfaces with his method. Many other complete surfaces, periodic as well as with finite topology, were constructed by Karcher [Karcher 1989] and the first author [Große-Brauckmann 1993] with extensions of the conjugate surface method.

Lawson's conjugate surface method generates symmetric surfaces by planar reflection from a simply connected fundamental domain. For all our surfaces this domain is bounded by five planar arcs and depends on two parameters. On the other hand there are two period conditions to satisfy. To solve these two period problems rigorously is a serious problem for our domains (see 2), and this is the main reason why we must rely on a numerical method. All other steps in our existence program can be theoretically proved similar to the non-compact examples constructed in [Große-Brauckmann 1993].

In our present paper we complete the studies started with the three examples of our previous paper [Große-Brauckmann and Polthier 1996] in the sense that we determine maximal families of similar surfaces; the surfaces are isolated and the families are finite. Our three previous surfaces were chosen rather close to the degenerate spherical situation, i.e. with thin necks, so that existence could be expected from Kapouleas' work [Kapouleas 1991] but not predicted. Most surfaces we present now have large necks. Thus they are further away from Kapouleas' class of surfaces, and they are also numerically easier to deal with.

In a way made precise in Sect. 4.2 our surfaces can be characterized by an underlying graph consisting of edges and vertices. Spheres or $k$-fold necks are located at the vertices, and one Delaunay neck on each edge of the graph. Our CMC surfaces have the symmetry of the underlying graph. These symmetries are given by discrete subgroups 


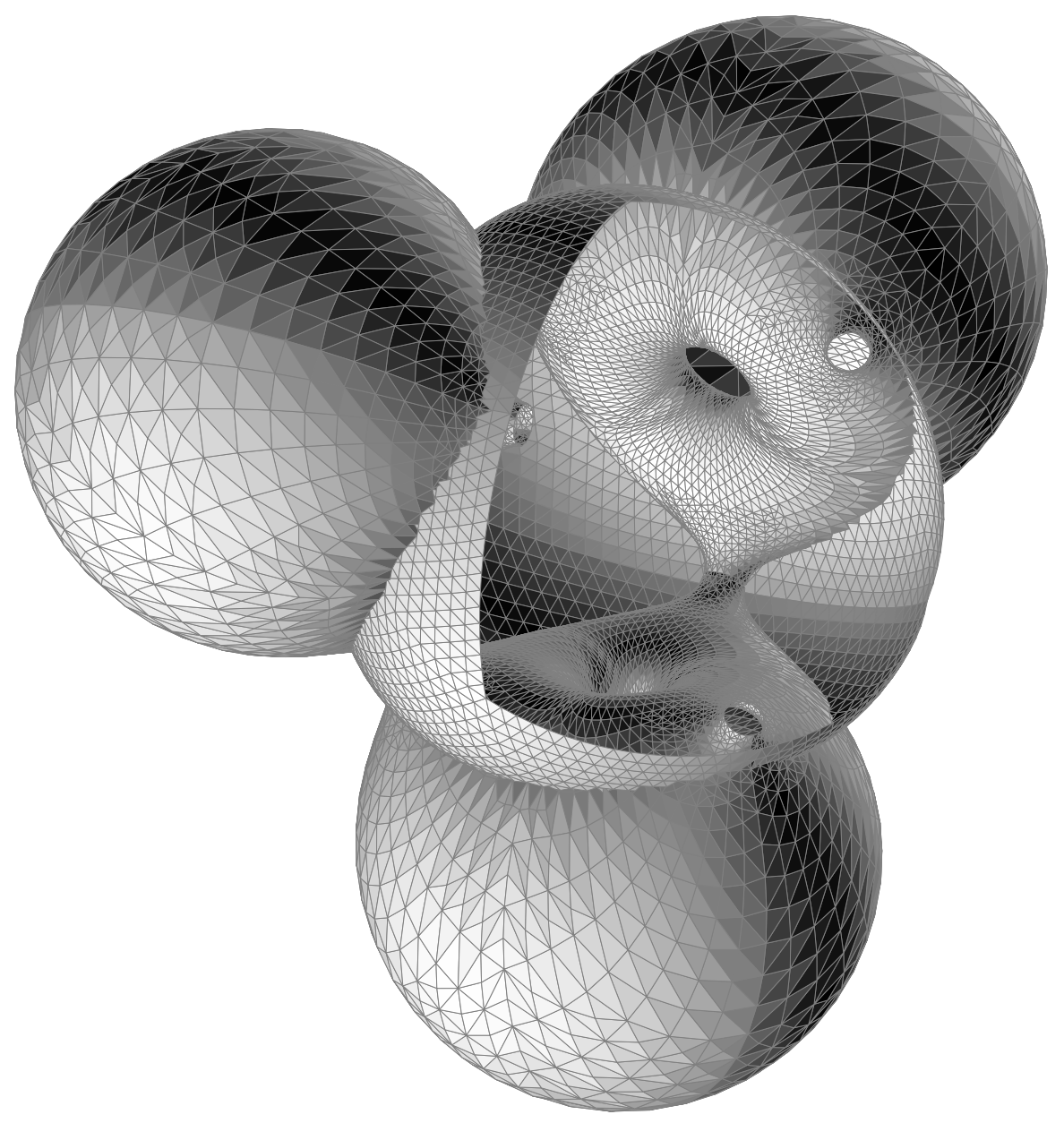

Figure 1. A surface of genus 6 with the symmetry of a tetrahedron. One bubble is removed. Six unduloidal necks join the four outer bubbles pairwise. The central bubble looks like a shell punctured in four points to connect it nodoidally to each outer bubble.

of $\mathrm{O}(3)$ generated by reflections: we have examples with dihedral symmetry and with the symmetry group of the Platonic polyhedra.

Which considerations guided our search for CMC surfaces, and which further surfaces can be expected to exist? There are necessary conditions to satisfy: most important is Kusner's balancing formula discussed in Section 4. We view this condition as a condition on the edge length of the underlying graph. Furthermore, the Delaunay surfaces and their dihedrally symmetric generalizations with $k$ ends (Section 5) indicate that further constraints than those given by the balancing formula are present. In particular a comparison with these surfaces leads to an explanation why our families only range over finitely many genera. 


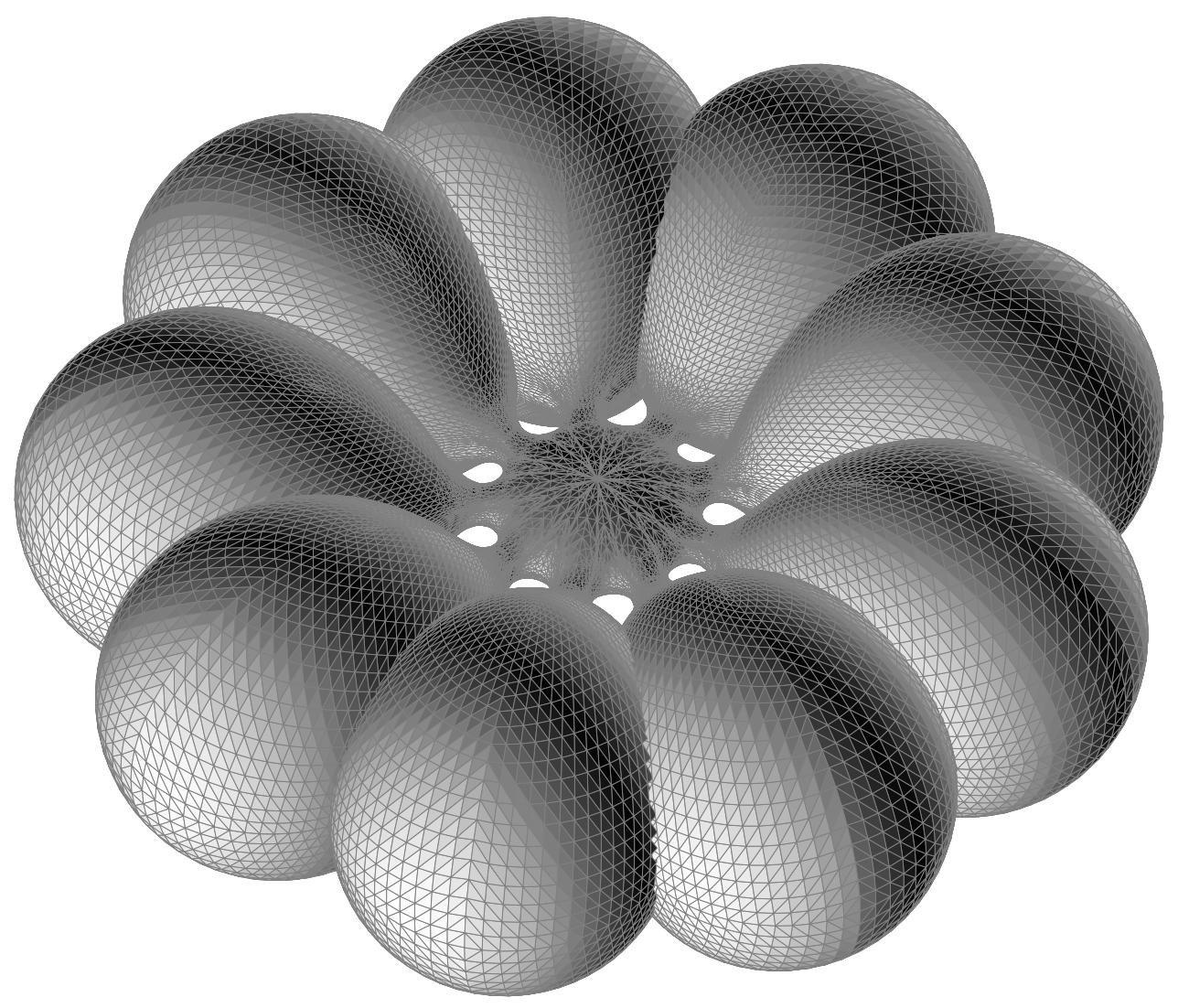

Figure 2. A dihedrally symmetric noidal surface of genus 9. We call the central 9-fold junction noidal because it is related to the minimal 9-noid. We find similar surfaces for all genera from 3 to 9 .

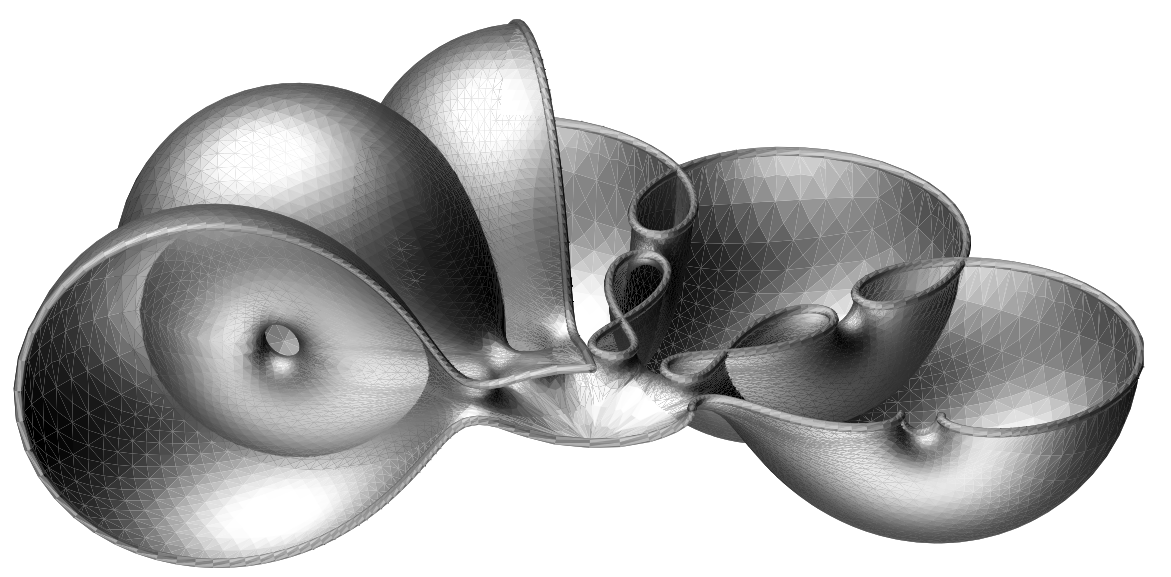

FiguRE 3. Part of the same surface with a view of the nodoidal necks connecting adjacent outer bubbles. The boundary is thickened with small tubes. 


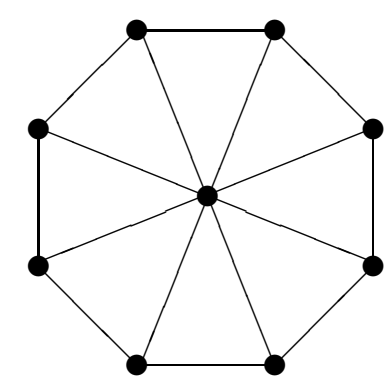

Figure 4. The underlying graph $G_{8}$ for the two genus 8 surfaces depicted in Figure 19 and 21. Vertices represent bubbles and edges necks.

The numerical algorithm of Oberknapp and Polthier generalizes an algorithm for discrete harmonic maps and minimal surfaces by Pinkall and Polthier [Pinkall and Polthier 1993]. There are two steps, one is to minimize area (in fact discrete Dirichlet energy) in $\mathbb{S}^{3}$, the other is to conjugate the discrete surface to a CMC surface in $\mathbb{R}^{3}$. The algorithm is implemented as part of the graphical environment GRAPE developed by the Sonderforschungsbereich 256 at the University of Bonn. The algorithm works with discrete data, and we cannot estimate how close the resulting polyhedral surfaces are to smooth CMC surfaces. Although we are confident that we correctly determined the range of genera for which our types of surfaces exist some care is appropriate with regard to the exact shape of our surfaces. We hope future proofs will support our experimental results.

\section{Results}

1.1. Dihedrally symmetric surfaces. In Table 1 we summarize the class of surfaces with genus $g$ and dihedral symmetry group $D_{g} \times \mathbb{Z}_{2}$. Slightly abusing notation we let this be the symmetry group of a planar regular $g$-gon considered as a subset of $\mathbb{R}^{3}$; the $\mathbb{Z}_{2}$-factor stands for reflection in the plane of the $g$-gon. $4 g$ copies of a fundamental domain such as the one shown in Fig. 6 combine to the entire compact surface. There are also $4 g$ cake piece shaped fundamental cells of $\mathbb{R}^{3}$ for these groups; as indicated by Fig. 6 such a cell does not contain an entire fundamental domain of the surface.

The soul of the surfaces is a planar graph $G_{g}$, consisting of a regular $g$-gon with $g$ additional edges (spokes) joining the midpoint to each vertex; Figure 4 shows $G_{8}$. By the balancing formula (see 4.1 below) $g$ must be at least 3 . The graph $G_{g}$ has only two independent lengths and we let its length quotient $q_{g}=2 \sin (\pi / g)$ be the quotient of the polygonal edge length over the length of the spokes. 


\begin{tabular}{|c|c|c|c|c|c|c|}
\hline \multirow{2}{*}{$\begin{array}{c}\text { genus } \\
g\end{array}$} & \multirow{2}{*}{ centre } & \multicolumn{2}{|c|}{ necks on } & \multirow{2}{*}{$\begin{array}{c}\text { length } \\
\text { quotient } q_{g}\end{array}$} & \multirow{2}{*}{$\begin{array}{l}\text { polygonal neck } \\
\text { circumference }\end{array}$} & \multirow{2}{*}{ Figure } \\
\hline & & spokes & polygon & & & \\
\hline 3 & \multirow{9}{*}{ spheroidal } & \multirow{3}{*}{\multicolumn{2}{|c|}{ nodoidal unduloidal }} & "1+0.73 & ( 0.66 & 16 \\
\hline 4 & & & & $1+0.41$ & 0.36 & 17 \\
\hline 5 & & & & $1+0.18$ & 0.16 & [GP1996] \\
\hline (6) & & (degenerate & 7 spheres) & 1 & $(0)$ & \\
\hline 7 & & \multirow{5}{*}{ unduloidal } & \multirow{5}{*}{ nodoidal } & $1-0.13$ & \multicolumn{2}{|c|}{ not studied } \\
\hline 8 & & & & $1-0.23$ & 0.23 & 19 \\
\hline 9 & & & & $1-0.32$ & 0.41 & - \\
\hline 10 & & & & $1-0.61$ & 0.63 & - \\
\hline$(\geq 11)$ & & & & \multicolumn{3}{|c|}{ surfaces do not exist } \\
\hline 3 & \multirow{8}{*}{ noidal } & \multirow{8}{*}{ unduloidal } & \multirow{8}{*}{ nodoidal } & $2(1-0.13)$ & 0.07 & $\overline{\text { [GP1996] }}$ \\
\hline 4 & & & & $2(1-0.29)$ & 0.21 & 18 \\
\hline 5 & & & & $2(1-0.41)$ & 0.31 & - \\
\hline 6 & & & & $2(1-0.5)$ & \multirow{2}{*}{\multicolumn{2}{|c|}{$\begin{array}{l}\text { not studied } \\
\text { not studied }\end{array}$}} \\
\hline 7 & & & & $2(1-0.57)$ & & \\
\hline 8 & & & & $2(1-0.62)$ & 0.65 & 21 \\
\hline 9 & & & & $2(1-0.66)$ & 0.71 & 2 \\
\hline$(\geq 10)$ & & & & \multicolumn{3}{|c|}{$\begin{array}{ll}\text { surfaces do not exist }\end{array}$} \\
\hline
\end{tabular}

TABLE 1. Surfaces with dihedral symmetry $D_{g} \times \mathbb{Z}_{2}$, graph $G_{g}$ and at most one neck per edge of the graph. [GP1996] stands for our previous paper [Große-Brauckmann and Polthier 1996].

For certain genera we obtain two different surfaces. These pairs are most clearly distinguished by the geometry of their centre in a way which is apparent from Fig. 19 and 21. Furthermore, the type which we call spheroidal has two different neck distributions which depend on the genus, in fact on the sign of $q_{g}-1$. In the following sections we will make the terminology used in Table 1 more precise and explain this fact.

By their symmetry the dihedrally symmetric surfaces have umbilics on the two points contained in the vertical axis of rotation. Using the Gauss-Bonnet formula it can be shown there are no further umbilics.

In the following sections we will explain why the experimental existence of certain surfaces implies the existence of others. Hence it was not necessary to carry out experiments for all surface candidates, and we marked surfaces we could skip with "not studied" in our tables. In Table 1 we also include a degenerate CMC surface consisting of seven spheres with symmetry $D_{6} \times \mathbb{Z}_{2}$. Since $q_{6}=1$ these spheres match in the sense that they touch tangentially on points of $G_{6}$.

Experimental Result 1. The fourteen dihedrally symmetric complete compact CMC surfaces listed in Table 1 exist. These are all $\mathrm{CMC}$ surfaces with graph $G_{g}$ and at most one neck per edge of the graph. 


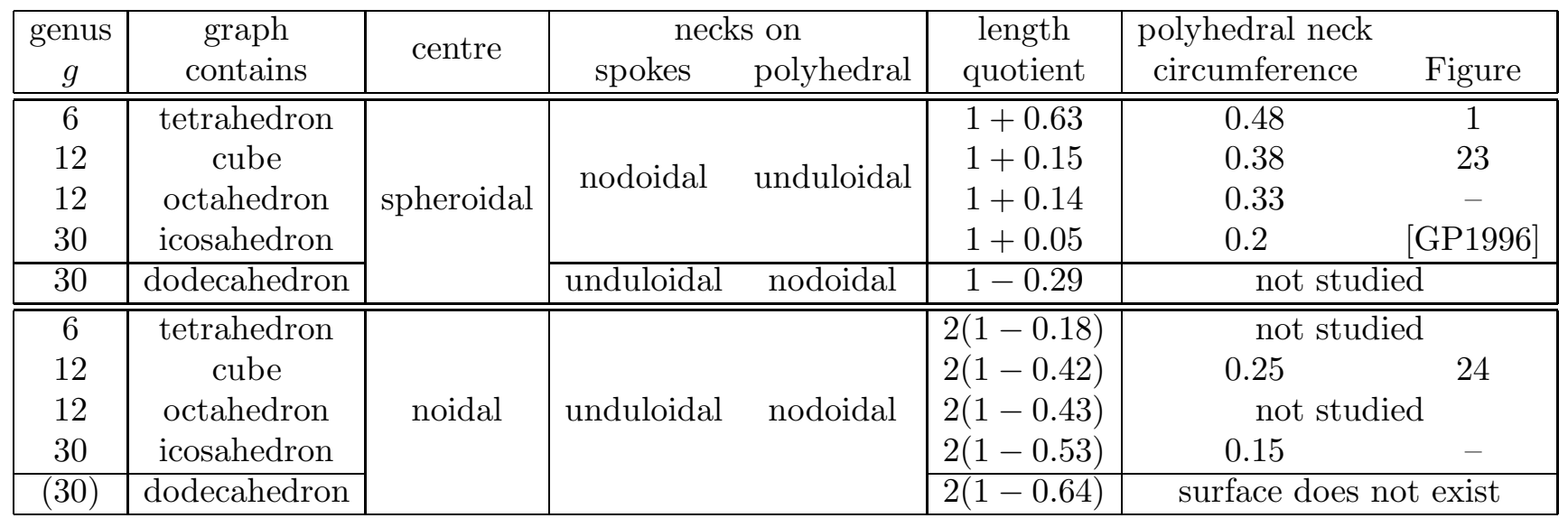

TABle 2. Surfaces with Platonic symmetry. The edges of the Platonic polyhedra with spokes to their centre form the graphs; the surfaces have at most one neck per edge.

We would like to remark that the graphs $G_{g}$ admit further surfaces with more than one neck per edge; in particular Kapouleas' construction [Kapouleas 1991] applies to some large number of bubbles. Also, in Sect. 7 we suggest further graphs which could lead to dihedrally symmetric surfaces.

1.2. Surfaces with Platonic symmetry. There are three singular discrete subgroups of $\mathrm{O}(3)$, given by the symmetry groups of the Platonic polyhedra. These groups are generated by reflections and we call them Platonic symmetry groups. The graph consists of the edge graph of a Platonic polyhedron with further edges (spokes) joining the vertices to the centre of the polyhedron. We obtain surfaces whose geometry is similar to the dihedrally symmetric surfaces.

Experimental Result 2. The nine complete compact CMC surfaces with Platonic symmetry listed in Table 2 exist. The surface with noidal centre and graph derived from the dodecahedron does not exist with one neck per edge.

As in the planar case we let the length quotient be the edge length of the polyhedron inscribed to the unit sphere. It is not the length quotient alone but also the combinatorics of the polyhedron that influence the polyhedral necksize listed in Table 2.

The genus of the surfaces with Platonic symmetry is the number of handles attached to the central sphere, that is the number of edges of the polyhedron. The outernmost point of each polyhedral bubble is umbillic, as well as further points on the central bubble. 


\section{The Conjugate surface COnstruction}

The conjugate surface construction for CMC surfaces generalizes a similar construction for minimal surfaces. Lawson established a local relation of CMC surfaces in $\mathbb{R}^{3}$ and spherical minimal surfaces in $\mathbb{S}^{3}$ :

Theorem 3. [Lawson 1970, p.364] ( $i$ ) For a simply connected minimal surface $M \subset$ $\mathbb{S}^{3}$ there exists an isometric $\mathrm{CMC}$ surface $\tilde{M} \subset \mathbb{R}^{3}$ and vice versa.

(ii) Furthermore, $M$ is bounded by a polygon $\Gamma$ of great circle arcs in $\mathbb{S}^{3}$ if and only if $\tilde{M}$ is bounded by geodesic curvature lines.

Let us suppose that a fundamental domain of a CMC surface with respect to a group of reflections is simply connected. Its boundary consists of piecewise planar geodesic curvature arcs. Thus by $(i i)$ the Plateau solution to a suitable great circle polygon in $\mathbb{S}^{3}$ can produce such a fundamental CMC domain, and this domain can then be reflected to a complete CMC surface. Which spherical polygon do we have to take? The angles $\pi /(k+1)$ (with $k \in \mathbb{N}$ ) at the vertices of the fundamental domain and the position of the normal at the vertices are needed to prescribe all angles of the spherical polygon. These data are immediate from the symmetry type of the fundamental $\mathrm{CMC}$ domain and determine the spherical polygon up to its lengths. In general a polygon with fixed angles and $n$ edges has $n-3$ free parameters for

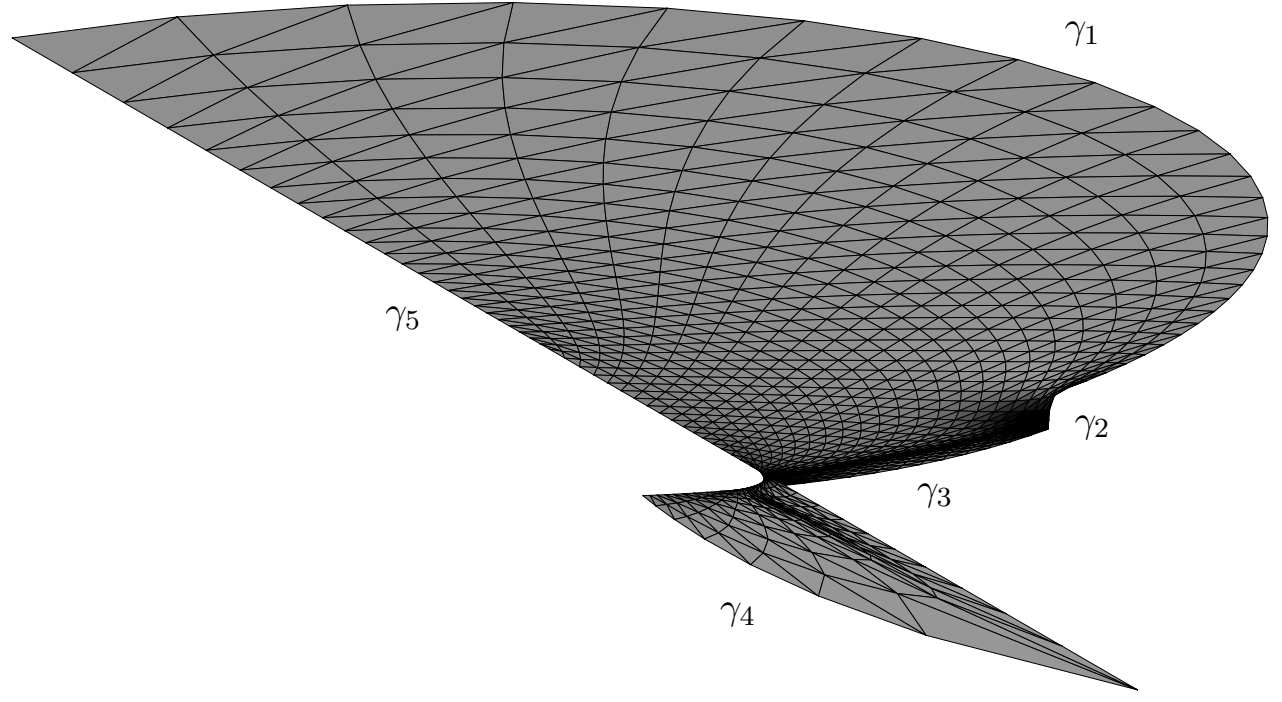

FiguRE 5. The fundamental domain for the spheroidal surface of genus 8 (see Figure 19). A polygon of five great circle arcs in $\mathbb{S}^{3}$ bounds the minimal surface patch. The patch is close to a great sphere $\mathbb{S}^{2} \subset \mathbb{S}^{3}$, or, in the chosen stereographic projection, close to a plane. There are two helicoidal regions, one connecting the triangle to the two-gon, the other in a neighbourhood of $\gamma_{2}$. 
the lengths. Not all lengths can be prescribed to any given value though. The set of lengths attained for a given set of angles can be determined using formulas of spherical trigonometry. The construction is explained in more detail in [Lawson 1970], [Karcher 1989], [Große-Brauckmann 1993].

For two reasons the conjugate surface construction will only lead to sufficiently symmetric CMC surfaces: first, the fundamental domain must be simply connected; second, the theoretical or numerical Plateau solution we take is a stable minimizer, and thus the fundamental domain must be small enough to be stable (see [Große-Brauckmann and Polthier $4.2])$.

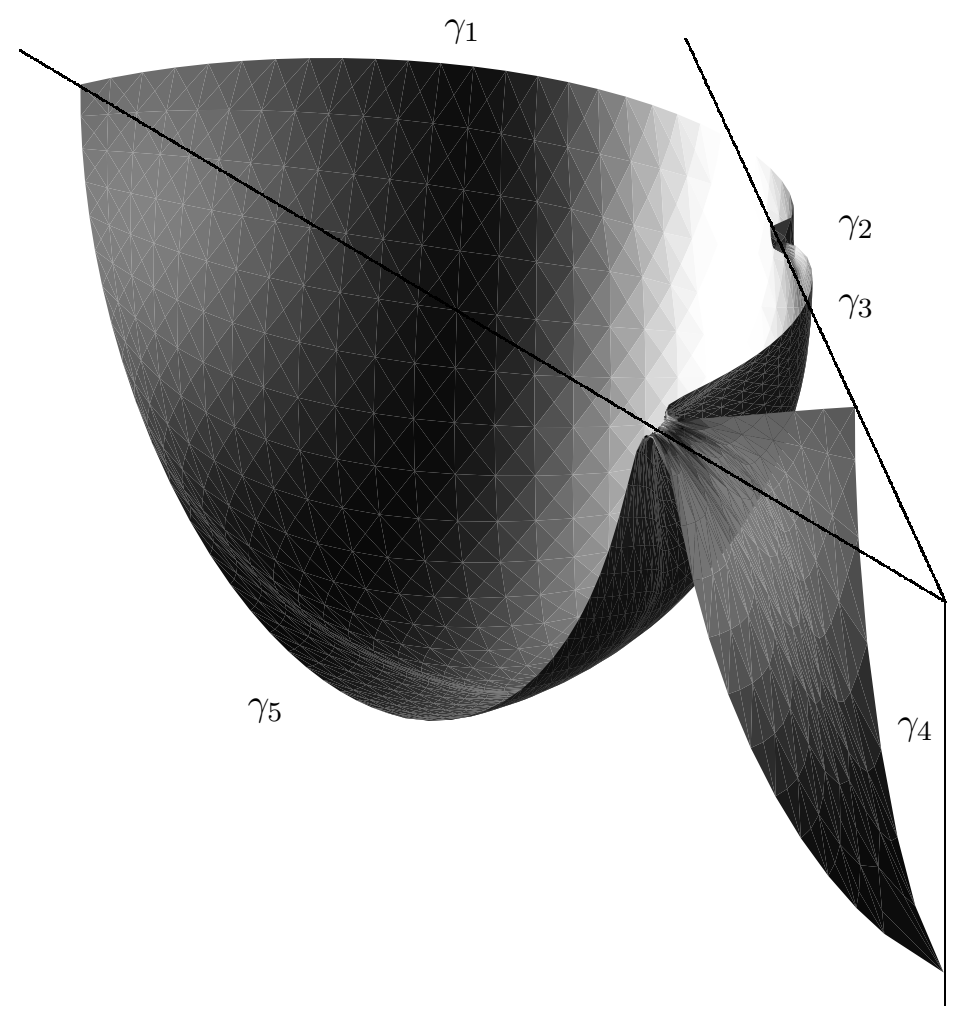

Figure 6. The isometric conjugate CMC patch. Its five boundary arcs are contained in three different planes that meet pairwise in the lines shown. Thirtytwo reflected copies generate the compact surface depicted in Figure 19. The almost-planar regions of the previous figure give spherical regions whilst the helicoidal regions result in necks. These can be nodoidal (at $\gamma_{2}$ ), or unduloidal (in between $\gamma_{3}$ and $\gamma_{5}$ ) depending on the sense of rotation of the helicoids.

Period problem. The spherical boundary polygons we consider in the present work are pentagons. Thus our fundamental CMC domains are bounded by five planar arcs which are contained in five planes. Two pairs of planes are parallel by construction, and only if they coincide the surface generated by reflection is compact - otherwise the surface will be doubly periodic. We solve this period problem by adjusting the 


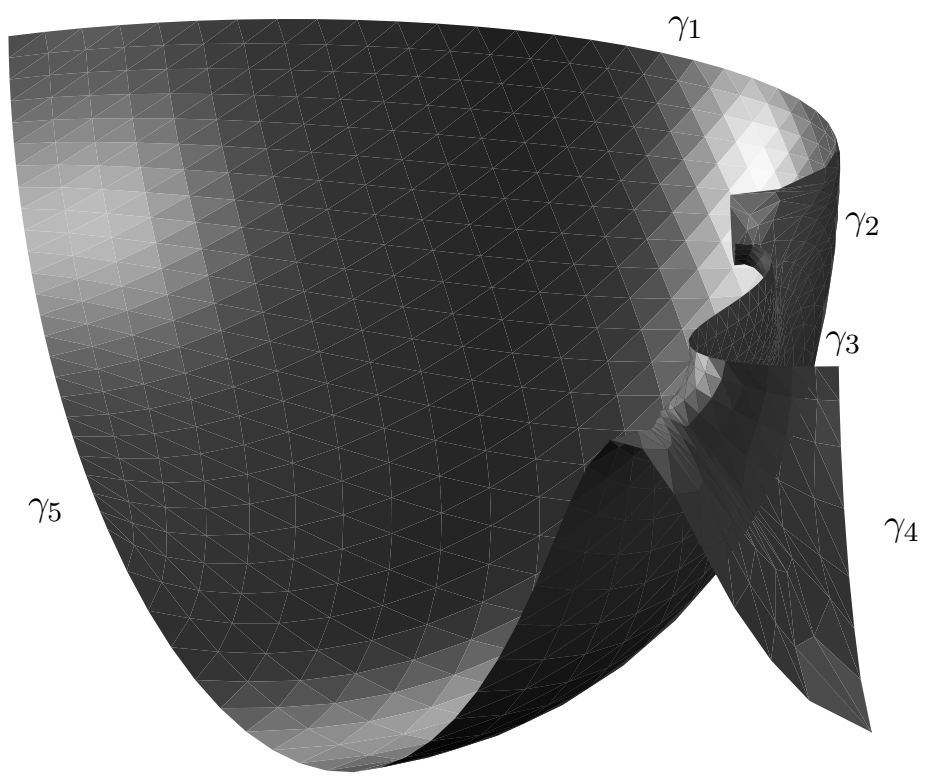

Figure 7. Period problem. The two parameter family of patches gives rise to two periods: one is given by the distance between the vertical planes containing $\gamma_{2}$ and $\gamma_{4}$, the other between the horizontal planes containing $\gamma_{1}$ and $\gamma_{3}$. Unlike the previous figure the patch shown has non-zero periods so that repeated reflection results in a doubly periodic CMC surface.

$5-3=2$ free parameters of the pentagon until the two pairs of planes coincide. Consequently our CMC surfaces are experimentally isolated.

In a more general sense all generators of the fundamental group give rise to periods. For our symmetric surfaces many of these periods agree; others, like the period of the unduloidal neck in Figure 7, are closed by symmetry. Thus the symmetry assumption reduces the number of different periods to two, regardless of the genus. Besides tori we do not know any compact surfaces that give rise to one period problem only. This makes two periods the simplest case to consider, while there are certainly many surfaces that pose three or more period problems.

To close the periods in a rigorous way one would have to give a loop in the parameter space so that the periods (viewed as a map to $\mathbb{R}^{2}$ ) can be estimated to have nonzero winding number about the origin. Then continuity of the family in its parameters would imply the existence of a surface with 0 periods. Continuity of the surfaces is experimentally observed, however, it is difficult to prove. The standard proof is to recover a surface as graph; it fails in the example of the spherical domain depicted in Fig. 5, since the two helicoidal regions are not graph with respect to any one direction. 


\section{THE ALGORITHM}

Fundamental pieces of CMC surfaces are rarely stable if they are considered as a solution to a free boundary value problem, see [Große-Brauckmann and Polthier 1996] for a discussion. Hence only sufficiently small fundamental domains can be obtained by minimization of area under a volume constraint. A more general approach suggested by $\mathrm{R}$. Kusner is to minimize the energy $\int(H-1)^{2}$; this can be done with Brakke's Surface Evolver [Brakke 1992] [Große-Brauckmann]. At present the compact surfaces of this paper seem out of reach for that approach.

Generalizing the algorithm of Pinkall and Polthier [Pinkall and Polthier 1993] for discrete (or polyhedral) harmonic maps and minimal surfaces, Oberknapp and the second author developed an algorithm that defines a discrete version of Lawson's conjugate surface construction. We refer to [Oberknapp and Polthier] and only point out two main ideas here.

Instead of minimizing the area functional the algorithm iterates the minimization of Dirichlet energy for discrete maps between discrete surfaces in $\mathbb{S}^{3}$ and produces a sequence of harmonic maps. Their images converge rapidly to a discrete minimal surface in $\mathbb{S}^{3}$ provided no triangles degenerate. In a second step the algorithm defines a conjugation method for discrete harmonic maps similar to the conjugation of smooth harmonic maps. Applying the conjugation to the above sequence of discrete harmonic maps produces a sequence of so-called 'conjugate' harmonic maps which map discrete surfaces in $\mathbb{S}^{3}$ to discrete surfaces in $\mathbb{R}^{3}$. The conjugation is exact on the discrete level. This fact is especially remarkable since in the smooth case the conjugation process uses $C^{1}$ information of the spherical minimal surface, which is of course not directly available for polyhedral surfaces. The images of the discrete conjugate harmonic maps converge to a discrete CMC surface in $\mathbb{R}^{3}$. An amazing result of the algorithm is that the boundary behaviour of the smooth case (Thm. 3(ii)) if fulfilled exactly by the discrete surfaces.

The resulting polyhedral surface is polygonal and not triangular. An interesting open problem is to give a discrete variational definition of "discrete CMC" for the above polygonal surfaces. Let us point out just one problem: a non-flat polygon can be filled in with surfaces in many ways, and thus the volume of a polygonal complex is not immediate. Since a variational characterization requires the notion of area and volume, it depends on the choice of surface. However, the characterizing property of the discrete surfaces the algorithm produces is that their spherical conjugates are discrete minimal surfaces in the sense that any variations of the vertices in $\mathbb{S}^{3}$ do not decrease area; this means Lawson's Theorem 3 can be taken as the definition of the discrete constant mean curvature, as could be done in the smooth case as well.

To apply the algorithm amounts to the following steps. 
- Determine the angular information of the desired CMC patch with $n$ planar geodesic boundary arcs. Guess $n$ edge lengths and fix $n-3$ of these. Taking the remaining three lengths as an initial condition, a rootfinder then finds a closed spherical polygon $\Gamma$ with $n$ great circle arcs as boundary.

- Solve the Plateau problem for $\Gamma$. In our examples the Gauß curvature varies considerably within a patch. Therefore interactive local refinement of the triangulation is necessary in regions of high curvature.

- The conjugation algorithm transforms a discrete minimal surface in $\mathbb{S}^{3}$ into a euclidean CMC surface.

- Check periods of the resulting CMC surface. If necessary repeat the previous steps with a different set of $n-3$ fixed initial lengths.

The algorithm can also give information on non-existence: when we try to adjust the $n-3$ lengths to obtain a surface with vanishing periods it can happen that we leave the range of existence for the boundary polygons.

The periods depend on the triangulation. Experiments described in [Oberknapp and Polthier] have shown this dependence to be surprisingly weak. Even so, we took care to adaptively triangulate those surface for which the period problem leads to polygons close to the boundary of existence.

\section{ForCES AND BALANCED GRAPHS}

4.1. Balancing of forces. Kusner's balancing formula gives a necessary condition on $\mathrm{CMC}$ surfaces derived from the first variation formula for a CMC surface; see e.g. [Korevaar et al. 1992]. The formula applies in general to 1-cycles $\gamma$ contained in a CMC surface $M$ and their bounding 2-chains $D$. Here, we have in mind that $\gamma$ is a curve running once around a neck and $D$ is a disk capping the neck. Considering all necks attached to a bubble then gives a condition at each bubble of a CMC surface.

To state the formula, let us assume that a set $B \subset \mathbb{R}^{3}$ (we think of a bubble) is bounded by an embedded subset $\Omega$ of the CMC surface $M$ as well as a finite number of disjoint disks $D_{i} \subset \mathbb{R}^{3}$. Each disk $D_{i}$ is in turn bounded by a circle $\gamma_{i} \subset \partial \Omega$. We choose exterior normals $\nu_{i}$ to $D_{i}$ and exterior conormals $\eta_{i}$ to $\gamma_{i}$, i.e. $\eta_{i}$ is a normal to $\gamma_{i}$ tangent to $\Omega$. Then the force associated to the neck is the vector

$$
W_{i}=\int_{\gamma_{i}} \eta_{i}-2 \int_{D_{i}} \nu_{i}
$$

The force can be shown to depend only on the homology class of $\gamma_{i}$. The balancing formula for $B$ now states that the forces of the adjacent necks are in equilibrium,

$$
\sum_{i} W_{i}=0
$$




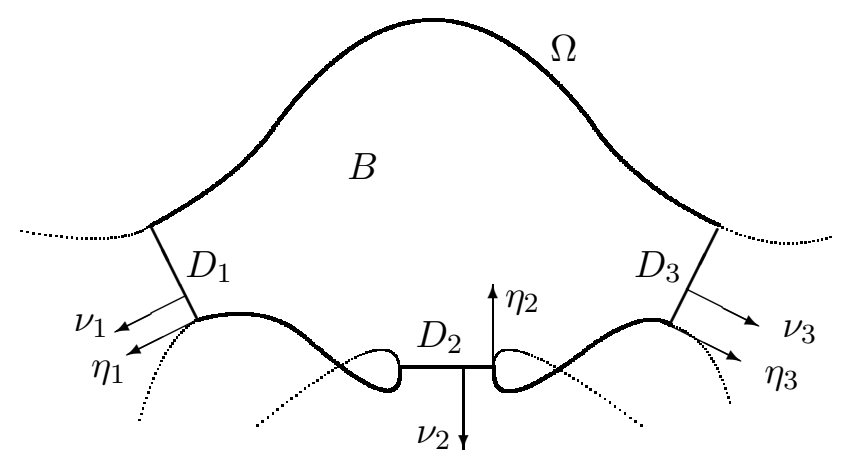

FiguRE 8. Notation for the balancing formula. For the bubble B the pull arising from the two lateral unduloidal necks is balanced by the force of the bottom nodoidal neck pushing the bubble upwards.

If a disk $D$ is contained in a plane then its normal is constant and $\int_{D} \nu=\operatorname{area}(D) \nu$. Moreover, if the plane is a symmetry plane for the surface then the conormal is also constant and $\int_{\gamma} \eta=\operatorname{length}(\gamma) \eta$ where $\eta= \pm \nu$. Thus we can express $W$ in the form

$$
W=( \pm \operatorname{length}(\gamma)-2 \operatorname{area}(D)) \nu
$$

4.2. Balanced graphs. An edge graph can be associated to our surfaces and more general to a class of (not necessarily compact) CMC surfaces [Kusner 1991]. This class includes surfaces arising from special constructions, namely the symmetric Delaunaylike surfaces of Kapouleas or the first author, but excludes tori for instance. The picture to keep in mind is that each vertex of the graph represents a bubble, and each edge a single neck or a piece of a Delaunay surface comprising several necks. The graph is a topological retract of the surface and finite for compact CMC surfaces. Figure 4 gives an example.

Each edge of the graph associated to a neck is taken to be parallel to its force vector. Kusner [Kusner 1991] chooses the line extending the edge as follows (Kapouleas' choice is different unless the surface is sufficiently symmetric). The homology class of a neck is assigned a torque $R_{i}(a)=\int_{\gamma_{i}} \eta \times(x-a)-2 \int_{D_{i}} \nu \times(x-a) \in \mathbb{R}^{3}$ which for given coordinates $x$ depends on $a \in \mathbb{R}^{3}$. Then it can be checked that $\left|R_{i}(a)\right|$ attains its minimum on a line $\left\{a_{0}+s W_{i} \mid s \in \mathbb{R}\right\}$; this is the line we want to define.

In general the set of these lines need not match in vertices, but in our case they do so by symmetry. Restricting the lines to the portion in between these intersection points gives a closed graph. Let us note that there is only one property of our graphs which is not immediate from symmetry: this is the length of the radial edges of our graphs. Thus scaling describes the only degree of freedom, and the minimization of torque in the above definition determines it. 

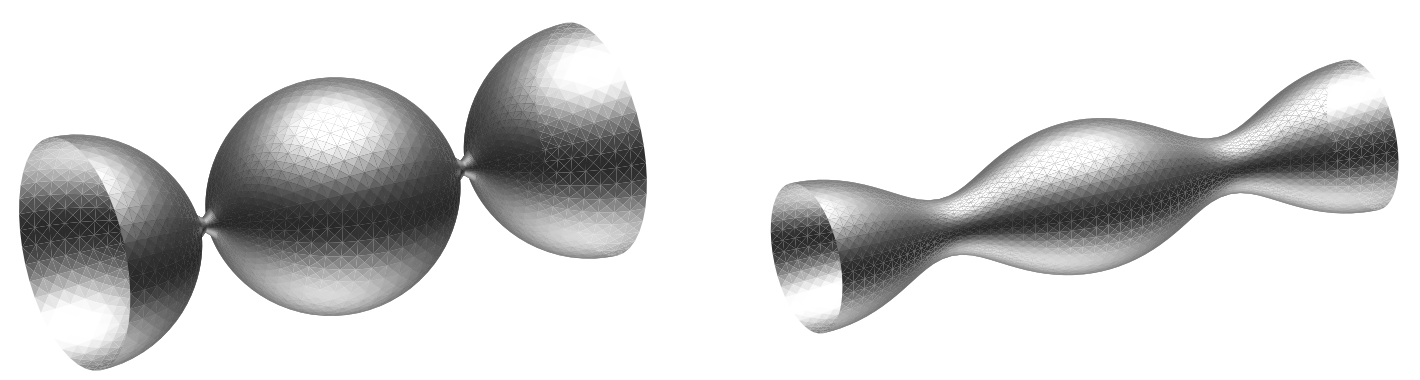

Figure 9. Two necks of two different Delaunay unduloids: As the unduloids deform from a chain of spheres to a cylinder their period increases from 2 to $\pi$.

For a closed graph there is an elegant way to express the balancing property. Let us label each edge of the graph associated to a CMC surface by a weight $w_{i} \in \mathbb{R}$ of the corresponding neck. We define $w_{i}$ by $\left|w_{i}\right|=\left|W_{i}\right|$ and let its sign be positive if the force is outward (when calculated for the bubble at a bounding vertex), or negative, if it is inward. Note that viewed from the opposite vertex the normals $\eta$ and $\nu$ change sign, so that inward and outward are well-defined. We will see below that by this definition unduloidal necks are assigned positive and nodoidal necks negative weights. Then for a given vertex the emanating edges $e_{i}$ considered as outward directed vectors can be used to state the balancing formula in the form $\sum w_{i} e_{i} /\left|e_{i}\right|=0$. A weighted graph with this property is called balanced.

¿From balancing many geometric properties of the graph follow. For instance if only two edges emanate from a vertex they must form a straight line, and we can omit the vertex. Thus the valence of each vertex can be assumed to be at least 3 .

\section{COMPARISION CMC SURFACES AND PRINCIPLES FOR EXISTENCE}

We discuss two classes of non-compact surfaces which serve for us as comparison surfaces. We present these surfaces first and then draw conclusions in the form of heuristic principles for existence.

5.1. Delaunay surfaces. The Delaunay surfaces are the non-compact CMC surfaces of revolution. A meridian is generated by the trace which the focus of an ellipse or hyperbola makes when these conical sections are rolled along a line. There are embedded unduloids and immersed nodoids. When normalized to mean curvature 1 each of them forms a one-parameter family of simply periodic surfaces.

The unduloid family ranges in between the cylinder and a degenerate CMC surface, a string of spheres. One choice of parameter is the extreme radius of the meridian: the minimum is $r$ and the maximum $1-r$ for the unduloids, with $r$ running from 0 

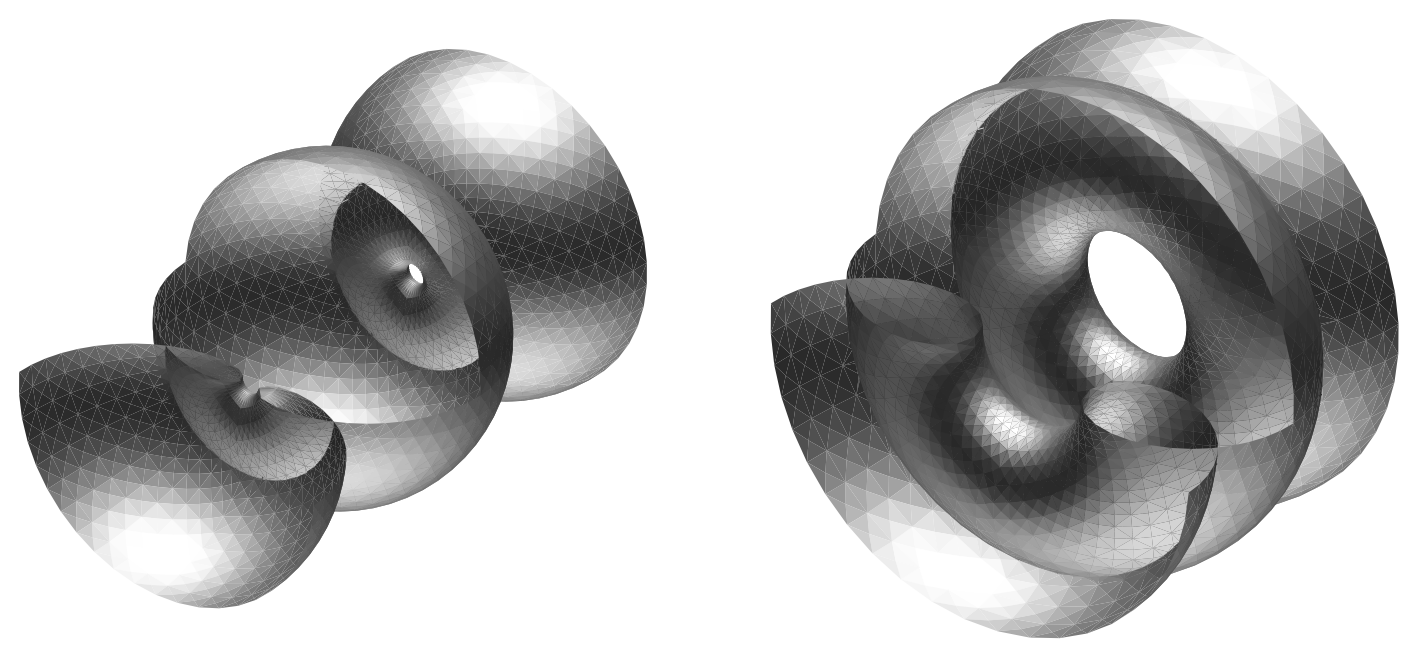

Figure 10. Two Delaunay nodoid necks (only the bottom half of the first shown): as the nodoids increase in diameter the period decreases from 2 to 0 .

(string of spheres) to $1 / 2$ (cylinder). A different choice of parameter is the weight. For a neck of an unduloid (3) gives the weight

$$
w_{U}=\operatorname{length}(\gamma)-2 \operatorname{area}(D)=2 \pi r(1-r)>0,
$$

where $r$ is the radius of the circle $\gamma=\partial D$. Hence $w_{U}$ decreases from $\pi / 2$ for a cylinder to 0 for the spheres.

The family of nodoids can also be parameterized with the extreme radii: these are $r$ and $1+r$ with $r \in \mathbb{R}_{+}$. When $r$ tends to infinity the nodoids leave every fixed cylinder. The weights of nodoid necks

$$
w_{N}=-\operatorname{length}(\gamma)-2 \operatorname{area}(D)=-2 \pi r(1+r)<0
$$

range from 0 (string of spheres) to $-\infty$. The period decreases from 2 for the sphere limit to 0 when $r$ tends to infinity.

Figure 11 gives the 1-1 correspondence of Delaunay periods and weights. The period can be considered the edge length of the balanced graph of a Delaunay surface (with vertices located at each bubble).

5.2. Dihedrally symmetric $k$-unduloids. These surfaces with symmetry group $D_{k} \times \mathbb{Z}_{2}$ have $k$ ends whose asymptotic axes are contained in a plane and make an equal angle with one another. The dihedrally symmetric $k$-unduloids provide an example that not all weights satifying the balancing formula do actually occur. 


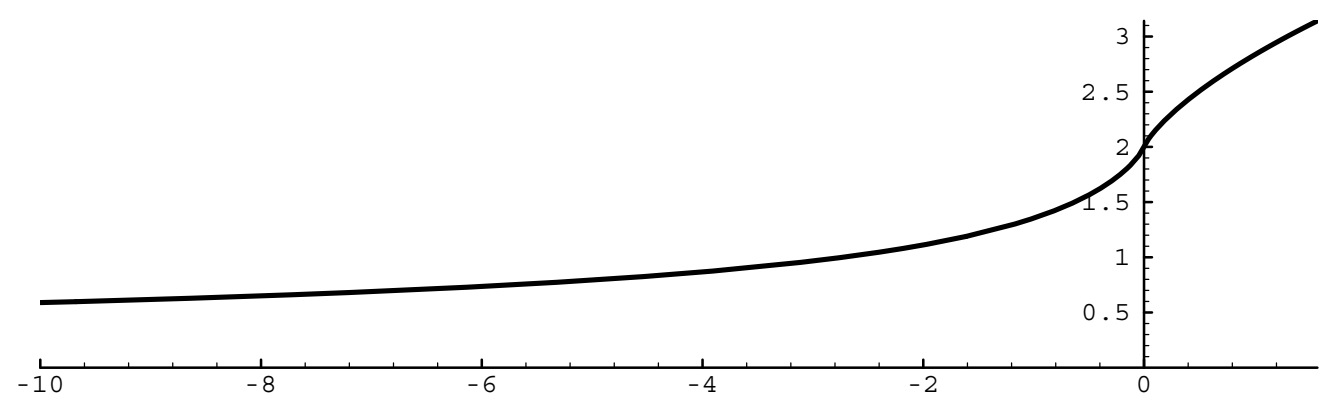

FiguRE 11. The period of the Delaunay surfaces in dependence on their weights. The immersed nodoids have negative weights $-\infty<w<$ 0 and periods tending to 0 with $w \rightarrow-\infty$. The embedded unduloids have positive weights $0<w<\pi / 2$, and periods ranging from 2 to $\pi$. The maximal weight $\pi / 2$ is attained by the cylinder. Weight 0 can be attributed to a degenerate Delaunay surface, a chain of unit spheres. Figure by courtesy of M. Heil.

Theorem 4. [Große-Brauckmann 1993] There exists a continuous one-parameter family of dihedrally symmetric $k$-unduloids for $k \geq 3$. Furthermore, the ends are asymptotic to unduloid ends and there is one dihedrally symmetric $k$-unduloid with ends of weight $w_{\max }(k):=2 \pi(k-1) / k^{2}$ and two for each weight $w$ with $0<w<w_{\max }(k)$.

How do the surfaces look like when we run through the entire one parameter family? The ends deform from rays of spheres (weight 0 ) to unduloids with some maximal necksize (given by $w_{\max }(k)$ ) and again back to spheres (weight 0 ). The central sphere which is present at one end of the family (Figure 12) shrinks away over the family. Thus, at the other end of the family the $k$ chains of spheres touch with their first sphere at the origin (the surface depicted in Figure 14 is still somewhat away from this situation). To distinguish the two surfaces of the same weight we call the former part of the family spheroidal and the latter noidal. The term noidal is justified by a blow up of the surfaces close to the degenerate limit. If this blow up is done at the right rate the centre of the surfaces converges to a dihedrally symmetric minimal surface with $k$ catenoid ends, the $k$-noid. This also holds for $k=2$ when the limit of small unduloid necks is a standard catenoid.

The maximal weight $w_{\max }(k)$ corresponds to a maximal asymptotic neck radius $1 / k$. Note that its decay in $k$ leaves enough room to attach the first necks of the ends at the central sphere; for a slower growth the necks would interfere and new neck shapes would have to develop for large $k$.

5.3. Construction Principles. The following heuristic principles guide our search for balanced graphs and their CMC surfaces.

(i) Weights and lengths are related similar to the Delaunay case (Figure 11). 


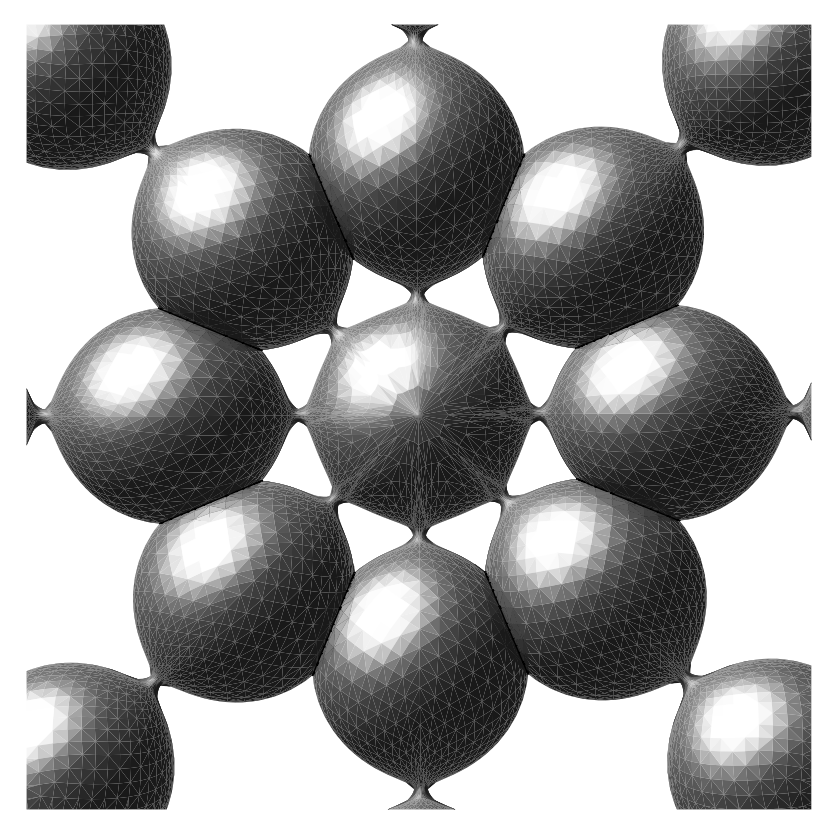

FIgURE 12. A (non-compact) dihedrally symmetric 8-unduloid with genus 0 and 8 unduloid ends. The first bubbles of the ends intersect. There is a one-parameter family of such surfaces. This surfaces is spheroidal in the sense that it has spherically shaped central bubble and unduloid ends of small forces. Existence of this surface is proven in [Große-Brauckmann 1993].

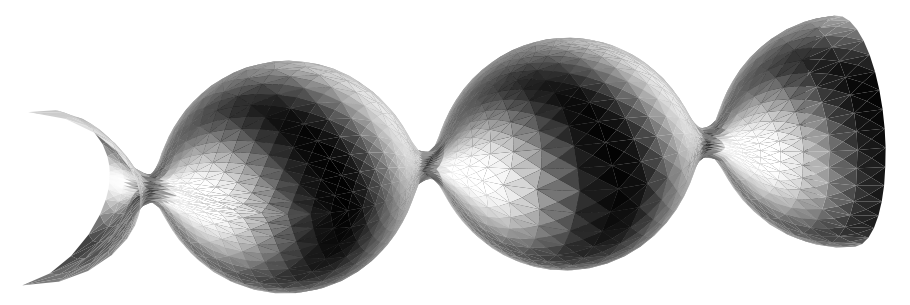

Figure 13. Sideview of one end; the first two and a half bubbles are shown.

(ii) An edge longer than 2 (but shorter than $\approx \pi$ ) is represented by an unduloid neck. If the length is larger than 4 we can take two unduloid necks enclosing an unduloid bubble, etc.

(iii) Edges of length less than 2 are represented by nodoidal necks. Again additional bubbles could be inserted for lengths less than integer multiples of 2 .

(iv) The weights resulting from the lengths must be compatible with the balancing formula (2).

We note that $(i i)$ and $($ iii $)$ are a consequence of $(i)$. We will also see that these two principles must be relaxed somewhat: in Section 6.2 we will represent edges 


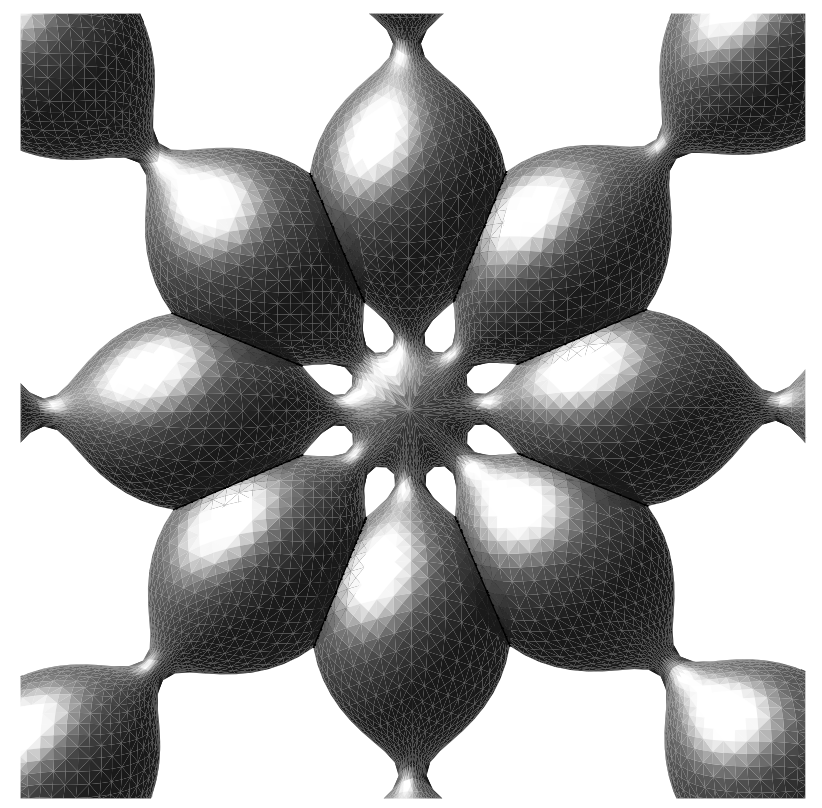

FIGURE 14. Another dihedrally symmetric 8-unduloid that with a noidally shaped central bubble. The weights of the ends are larger than those in Figure 12. As the weight tends to zero the middle junction of the surface shrinks to a point. Existence of the surface is proven in [Große-Brauckmann 1993].

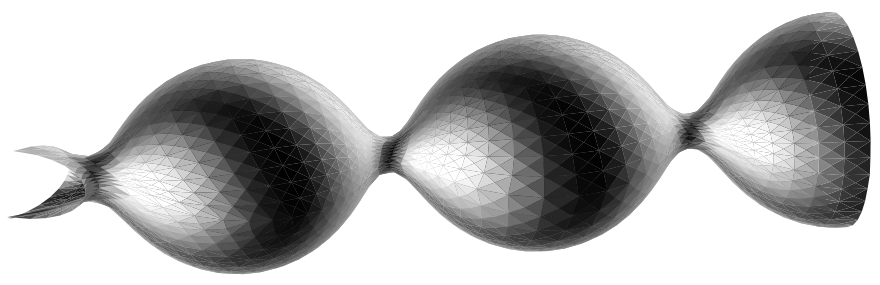

FiguRE 15. Sideview of one end.

longer than 1 by an unduloid neck. At each vertex the balancing condition (iv) imposes effectively one constraint on the weights and therefore on the lengths of the emanating edges.

Similar to the Delaunay comparison principle $(i)$ we want to include a comparison for dihedrally symmetric necks to our list.

$(v)$ The radial weight of a dihedrally symmetric $k$-fold neck is at most $\approx w_{\max }(k)$.

Without the symmetry assumption it is unclear which range of weights one should expect ([Große-Brauckmann and Kusner] [Große-Brauckmann and Polthier] discuss this problem for some examples). 
We draw an important conclusion. Suppose all edges at a vertex point into a halfspace. This holds e.g. on the exterior vertices of a finite graph. Then (iii) implies that both unduloidal and nodoidal necks should be present. By $(i)$ and $(i i)$ edges of lengths both smaller and larger than 2 should emanate from the vertex. Similar conditions could be formulated if we have in mind to place $k$ necks enclosing $k-1$ bubbles on an edge.

\section{FAMilies OF COMPACT SURFACES}

We now want to apply the five guiding principles of the preceding section to the graphs of our compact surfaces. No further constraints are present. Principle (iv), the balancing formula, implies that the valence of each vertex is at least 3 , so that the graph $G_{2}$ (see Sect. 1) does not arise from our construction.

We first consider the dihedrally symmetric case. For the midpoint of $G_{g}$ the balancing formula (2) is satisfied by symmetry. Indeed $g$ radial edges emanate from the central vertex and they all have the same (positive or negative) weight. For the outer vertices all edges point into a halfspace and, as pointed out in Section 5.3, the balancing formula implies that unduloidal and nodoidal necks should be present on the adjoining edges. Which distribution is appropriate depends on the length quotient $q_{g}$. The radial and polygonal edges enclose an angle $\pi / g$, so that by the balancing formula (2) the two polygonal weights $w_{P}(g)$ result in a radial weight $w_{R}(g)$

$$
w_{R}(g)=-2 \sin (\pi / g) w_{P}(g) .
$$

6.1. Dihedrally symmetric spheroidal surfaces. We view $w_{R}(g)$ and $w_{P}(g)$ as functions of the lengths of $G_{g}$, with values approximately given by the Delaunay weights. We look for a scaling of the graph $G_{g}$ such that the induced weigths $w_{R}(g)$ and $w_{P}(g)$ satisfy (5). For $g=6$ the degenerate weights $w_{R}=w_{P}=0$ satisfy this equation, and the corresponding scaling of $G_{6}$ with edge length 2 is the graph for the degenerate surface consting of 7 touching spheres.

For $3 \leq g \leq 5$ we have $q_{g}>1$. Thus there is a scaling of the graph $G_{g}$ with radial edges shorter than 2 and polygonal edges longer than 2. In view of principle (ii) and (iii) this suggests unduloidal necks on the polygonal edges and nodoidal necks on the spokes. Using the Delaunay comparison principle $(i)$ we see the length of the spokes must be approximately in the interval $\left(2 / q_{g}, 2\right)$ : the left endpoint corresponds to polygonal edge length 2 so that the right hand side of (5) vanishes, whereas at the right endpoint the left hand side of (5) vanishes. Thus the actual length can be viewed to be the result of an intermediate value problem for (5).

Note that for the same radial edgelength both $\sin (\pi / g)$ and, assuming principle $(i)$, $w_{P}(g)$ are larger for $g=3$ than for $g=4$ and 5 . Thus for (5) to hold the scaling of $G_{3}$ 


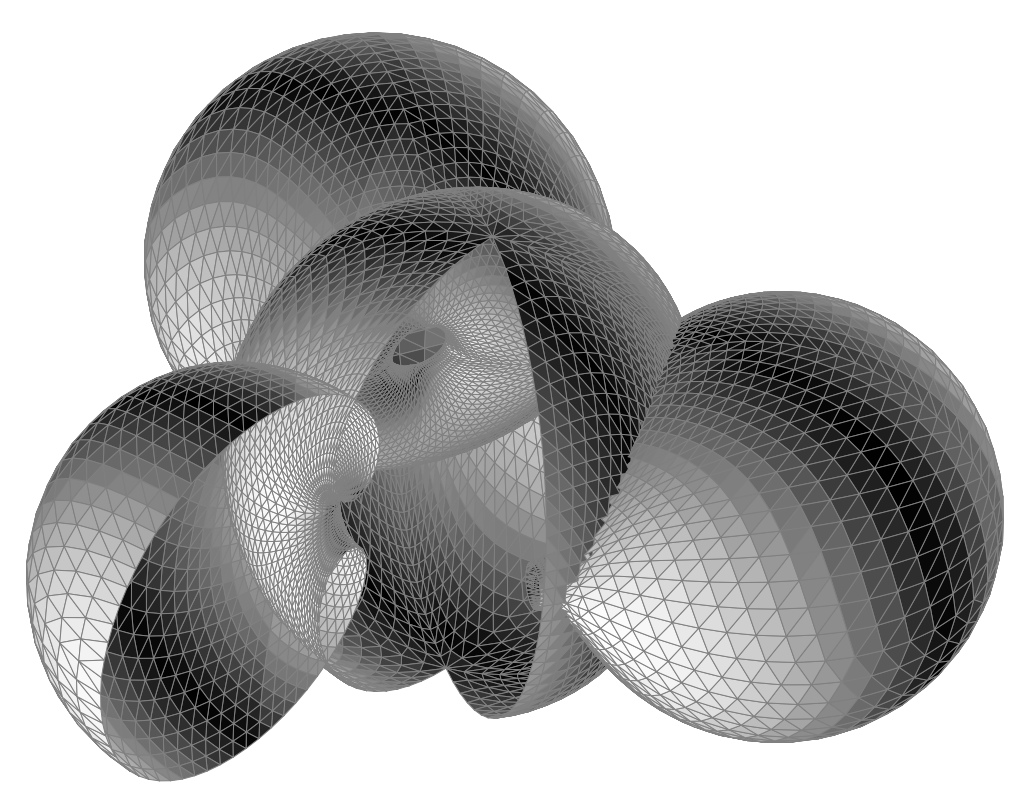

Figure 16. Spheroidal surface of genus 3. A sixth of the surface is removed. As the tetrahedral surface of Figure 1 the three unduloidal necks are contained in the central bubble (see the front end of the unduloidal bubble on the right).

must be smallest. This gives the genus 3 surface the largest radial necks in agreement with our experimental results.

If $g$ is greater than 6 then $q_{g}<1$ so that the polygonal edges of $G_{g}$ are shorter than the spokes. To be consistent with principle $(i i)$ and $(i i i)$ we need to flip unduloidal 


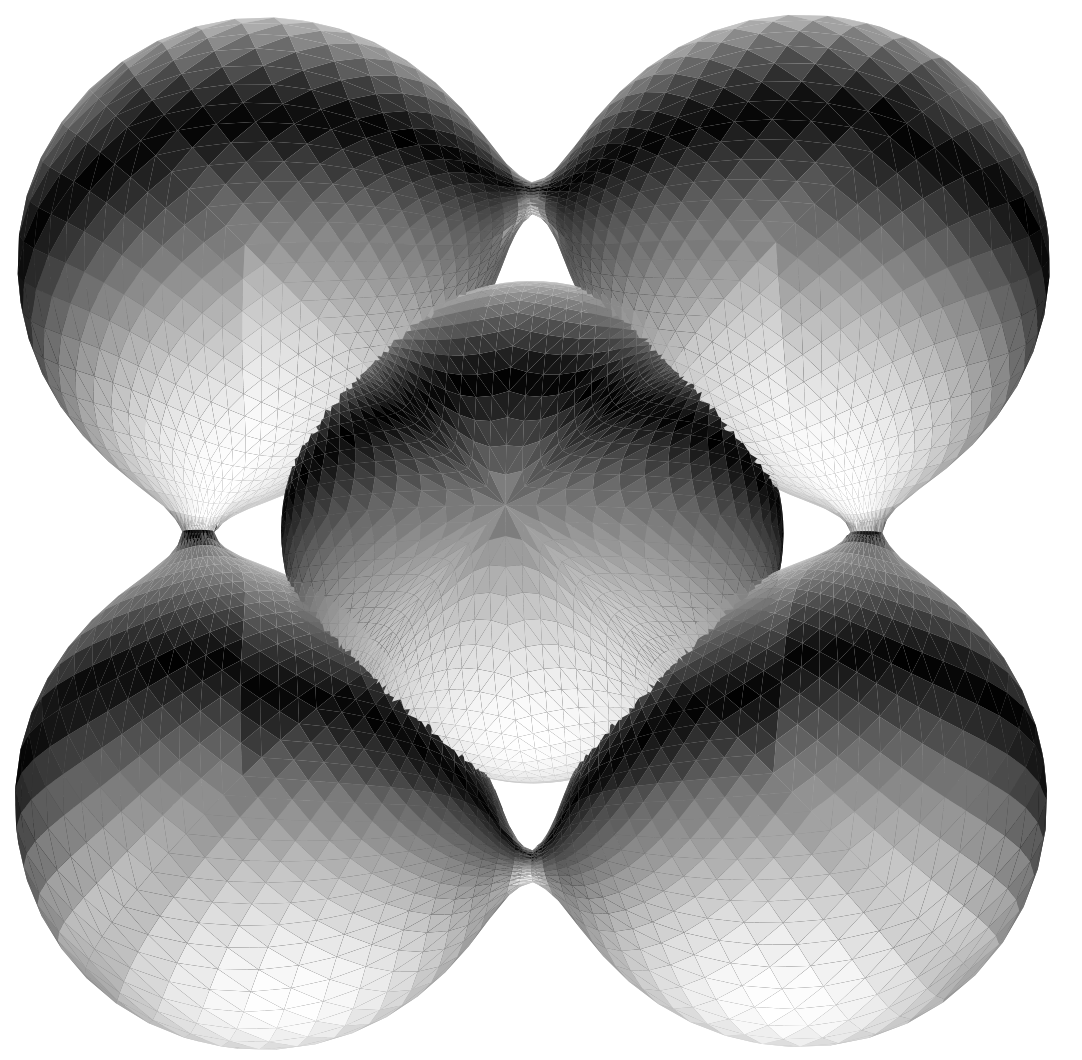

Figure 17. Spheroidal surface of genus 4. The outer bubbles are connected by unduloidal necks and in turn have nodoidal necks joining them to the central bubble. The outer bubbles look much like bent unduloids and, like these, they are flatter than the centre bubble.

and nodoidal necks compared to the previous family. An intermediate value argument similar to the above gives that $G_{g}$ with spokes of unit length has to be scaled with some factor in $\left(2,2 / q_{g}\right)$. We obtained existence for $g=8,9,10$ with increasing necksizes. For genus 7 the necks are thin and the surface is numerically harder to deal with our algorithm; on the other hand Kapouleas' theoretical existence result makes this surface most likely to exist. Therefore we skipped $g=7$.

6.2. Dihedrally symmetry noidal surfaces. Similar to the noidal and spheroidal dihedrally symmetric $k$-unduloids of the same weight (Sect. 5.2) we obtain a further set of CMC surfaces with the same graphs $G_{g}$. These have a noidal central bubble and nodoidal polygonal necks. For this type there is only "half a neck" on each spoke but still an entire neck on each polygonal edge. The critical length quotient is therefore $q_{g}=\frac{1}{1 / 2}=2$. The difference $2-q_{g}$ is always positive (for $g \geq 3$ ), and by principle $(i)$ we must have unduloidal spokes and nodoidal polygonal necks for all $g \geq 3$. 


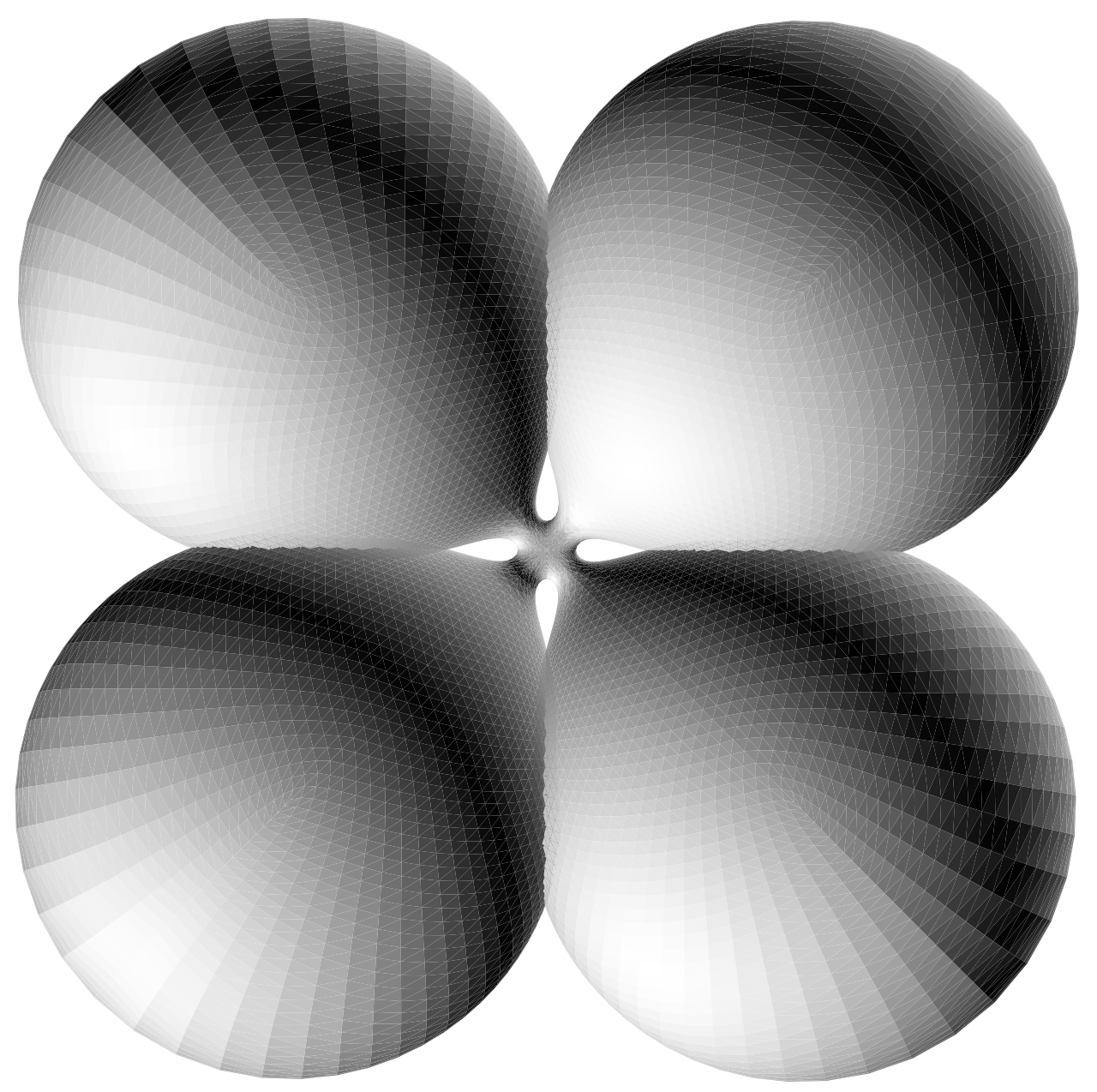

Figure 18. A noidal of genus 4 for which nodoidal and unduloidal necks are flipped relative to the previous surface. This surface has a central 4-noidal neck, the outer bubbles are connected with nodoid necks which are not visible in this view.

Our experiments covered genus 4, 5, 8, and 9; we skipped genus 6 and 7 because in view of principle $(v)$ existence is clear to us from the existence of the higher genera. In [Große-Brauckmann and Polthier 1996] we mentioned the numerical complications we faced when we tried to solve the period problem for the surface with $g=3$, so that we did not have enough experimental evidence to claim existence of the surface. However, in view of the existence of similar surfaces with larger genus (and larger necks) we do not doubt existence for genus 3 any more. This is in agreement with principle $(v)$ : for large, not for small necksizes, existence is problematic.

How do the necksizes of a spheroidal and noidal surface relate for the common genera 7 to 9 ? Clearly the scaling of the noidal graph is smaller. This makes the 


\begin{tabular}{|c|c|c|c|c|}
\hline $\begin{array}{c}\text { genus } \\
g\end{array}$ & $\begin{array}{c}\text { weight of a Delaunay } \\
\text { nodoid with period } 2 q_{g} \\
\text { (cf. Fig. 11) }\end{array}$ & $\begin{array}{c}\text { resulting } \\
\text { radial weight } \\
\text { assuming (5) }\end{array}$ & $\begin{array}{c}\text { approximate } \\
\text { experimental } \\
\text { radial weight }\end{array}$ & $\begin{array}{c}\text { max. weight } w_{\max }(k) \\
\text { of a dihedrally } \\
\text { symmetric } k \text {-unduloid }\end{array}$ \\
\hline \hline 7 & -0.23 & 0.20 & $(-)$ & 0.77 \\
8 & -0.57 & 0.43 & 0.18 & 0.69 \\
9 & -0.96 & 0.62 & 0.29 & 0.62 \\
10 & -1.2 & 0.87 & 0.43 & 0.57 \\
15 & -4.4 & 1.8 & - & 0.39 \\
20 & -8.6 & 16 & - & 0.30 \\
100 & -248 & 84 & - & 0.06 \\
500 & -6700 & \multicolumn{3}{|c}{} \\
\hline
\end{tabular}

TABLE 3. Weight comparison for the dihedrally symmetric surfaces

polygonal lengths of the noidal surfaces larger so that the nodoidal necks are larger. By (5) the unduloidal necks must be larger too.

6.3. Finiteness of the dihedrally symmetric families. The key to understanding the upper limit $g=10$ of the spheroidal family is to see how the radial weight grows in the genus. Unlike the spheroidal case for $g=3,4,5$ an estimate of the radial forces is not straightforward from (5); indeed with increasing genus $\sin (\pi / g)$ decreases, whereas the nodoidal weights $\left|w_{P}(g)\right|$ increase for shorter edges according to principle $(i)$. Hence it is not clear how the resulting weight $-2 \sin (\pi / g) w_{P}(g)$ depends on $g$; it could still be bounded for $g \rightarrow \infty$.

Let us give an estimate for $-2 \sin (\pi / g) w_{P}(g)$ based on the Delaunay comparison principle $(i)$. To simplify we assume that the scaling of the graph has spokes of length 2. This gives the polygonal edge a length $2 q_{g}=4 \sin (\pi / g)$, i.e. we consider one of the limiting cases of the intermediate value argument of 6.1 . In Table 3 we list the weight of a Delaunay nodoid with period $2 q_{g}$. The values of $w_{P}$ resulting from (5) are unbounded. However, from the Delaunay comparison we expect an upper bound for the weight and thus only finitely many compact surfaces of the considered type should exist.

More specifically, by principle $(v)$ the weights should be no larger than approximately $w_{\max }(k)$. This holds up to genus 9 , which is in rather good coincidence with our experimentally determined limiting genus 10 . We note that in fact the actual lengths of the graphs are larger, so that the weights are smaller than the estimate given in Table 3. For comparison we computed approximate experimental weights by assuming that the polyhedral neck crosssection is a circle of the length stated in Table 1, for which (4) gives the weight. The resulting weights are experimental evidence for $(v)$ : up to genus 10 they are well below $w_{\max }(10)$, but for genus 11 the weight expected from linear extrapolation would be larger than $w_{\max }(11)$. 
How does the limitation in genus appear in our experiments? We can still find fundamental domains for higher genus, but we cannot close the periods. The reason for this is that the range of existence for the boundary polygons is limited. This range can be determined using spherical trigonometric formulas. For genus 10 we are well away from the boundary of existence and for genus 11 the periods for existing domains are sufficiently large to give us confidence that smooth CMC surfaces also exist exactly up to (and including) 10.

We can also explain why the maximal genus 9 of the noidal surfaces is smaller than the maximal genus 10 of the spheroidal family. As we pointed out in 6.2 the necksizes of the noidal surfaces are larger than those of the spheroidal surfaces. Consequently the noidal surfaces reach the limiting weight $\approx w_{\max }(k)$ for a lower genus than the spheroidal ones.

6.4. Platonic symmetry. The value for the length quotient listed in Table 2 suggests four spheroidal surfaces with unduloidal polyhedral necks and nodoidal radial necks, and our experiments gave existence for all these cases. The dodecahedron has length quotient less than one and we expect a surface of genus 30 with 20 outer bubbles with polyhedral nodoidal necks. We did not investigate this surface but its existence seems very likely.

Similar to the dihedrally symmetric case there is a noidal family. The length quotient for the dodecahedral surface is so far away from 2 that the radial necks must be too large to exist - this explains why we could not close the periods for the dodecahedral surface. However, the isosahedral and cubic surface exist. In view of $(v)$ we are confident that this implies existence for the surfaces with smaller weights: these are the surfaces with tetrahedral and octahedral graph and symmetry.

\section{Further Classes}

We would like to mention a few classes of graphs that could admit similar compact CMC surfaces. We choose examples that are symmetric enough for our construction to be applicable. Fig. 26.1 shows a square whose opposite vertices are joined by two further edges (no vertex at the centre). Similarly, a regular $2 k$-gon $(k \geq 2)$ whose opposite vertices are joined gives a graph with dihedral symmetry $D_{2 k} \times \mathbb{Z}_{2}$; the corresponding CMC surfaces would have genus $k+1$. Fig. 26.2 shows a different way to connect the vertices of $G_{6}$ : while the spokes are kept every other hexagon point is joined. Similarly $g+1$ points $(g \geq 5)$ give a graph with symmetry $D_{g} \times \mathbb{Z}_{2}$, for candidate surfaces of genus $g$. Instead of every other also every third, fourth, etc. polygonal vertex could be joined, so that there are further similar families. In Fig. 26.3 the modification of $G_{6}$ is opposite: the polygonal edges of $G_{6}$ are kept, but there are two different vertices in the centre, each one joined to every other polygonal vertex. More generally, a $2 k$-gon gives a graph of symmetry $D_{2 k} \times \mathbb{Z}_{2}$ for surfaces 


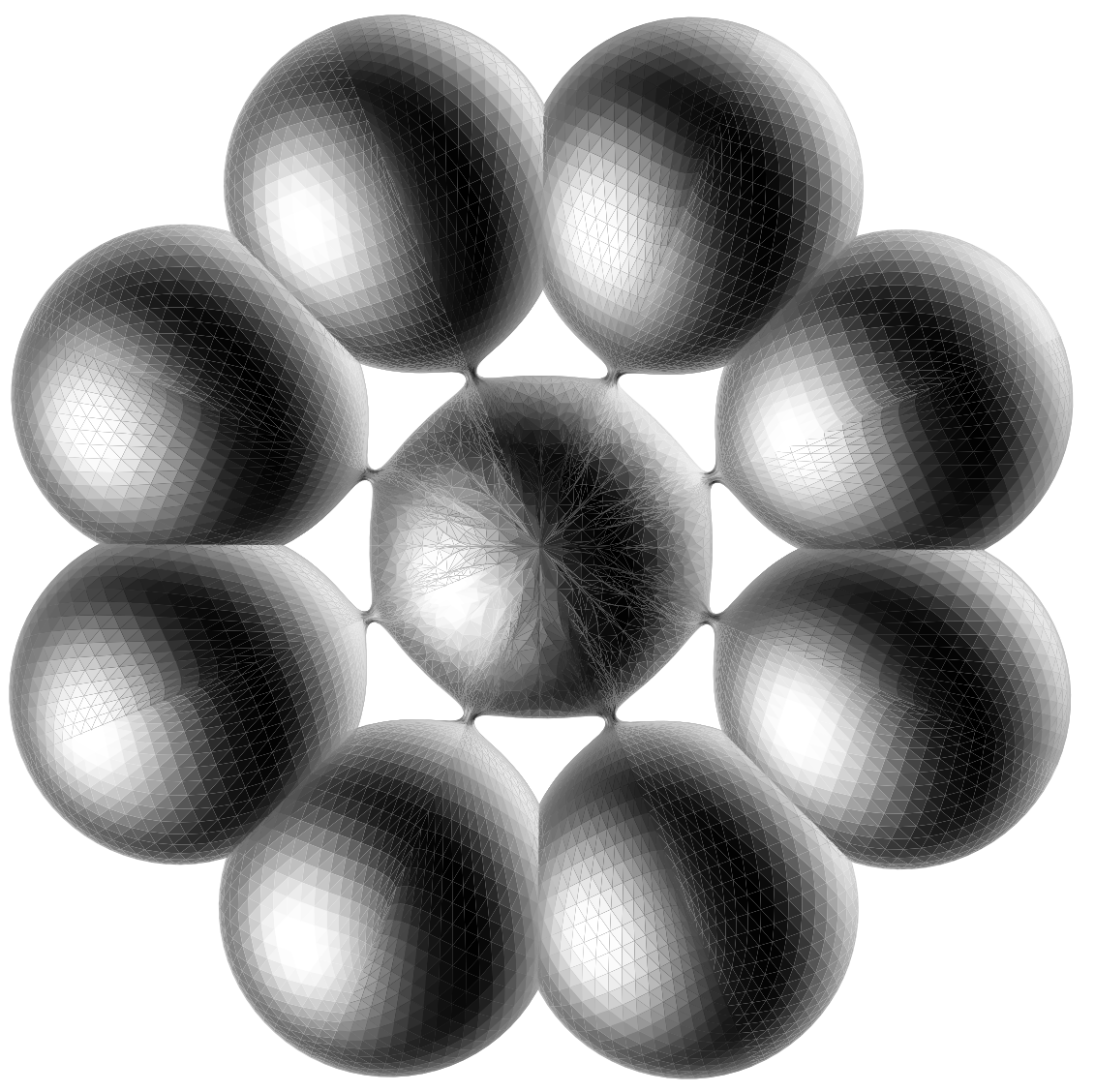

Figure 19. Spheroidal surface of genus 8. The outer bubbles are connected with nodoidal necks. The bubble in the middle is almost spherical. Compare to Figure 12.

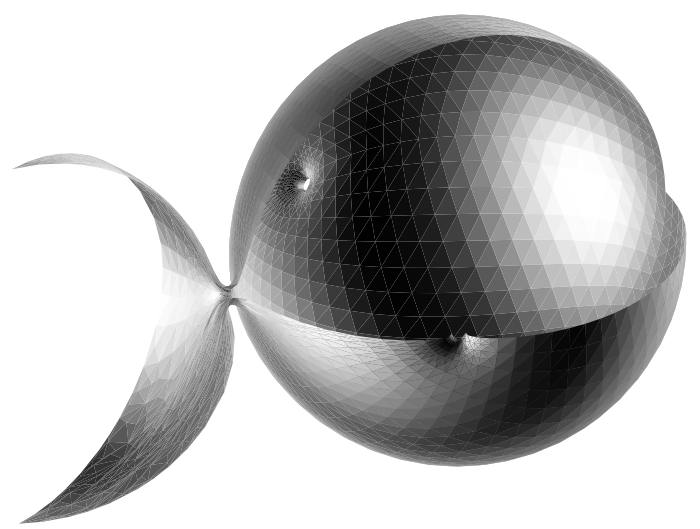

Figure 20. Three fundamental domains of the same surface give a view of the nodoidal necks connecting adjacent bubbles.

of genus $2 k-1$; again there are similar families with further central vertices. Other 


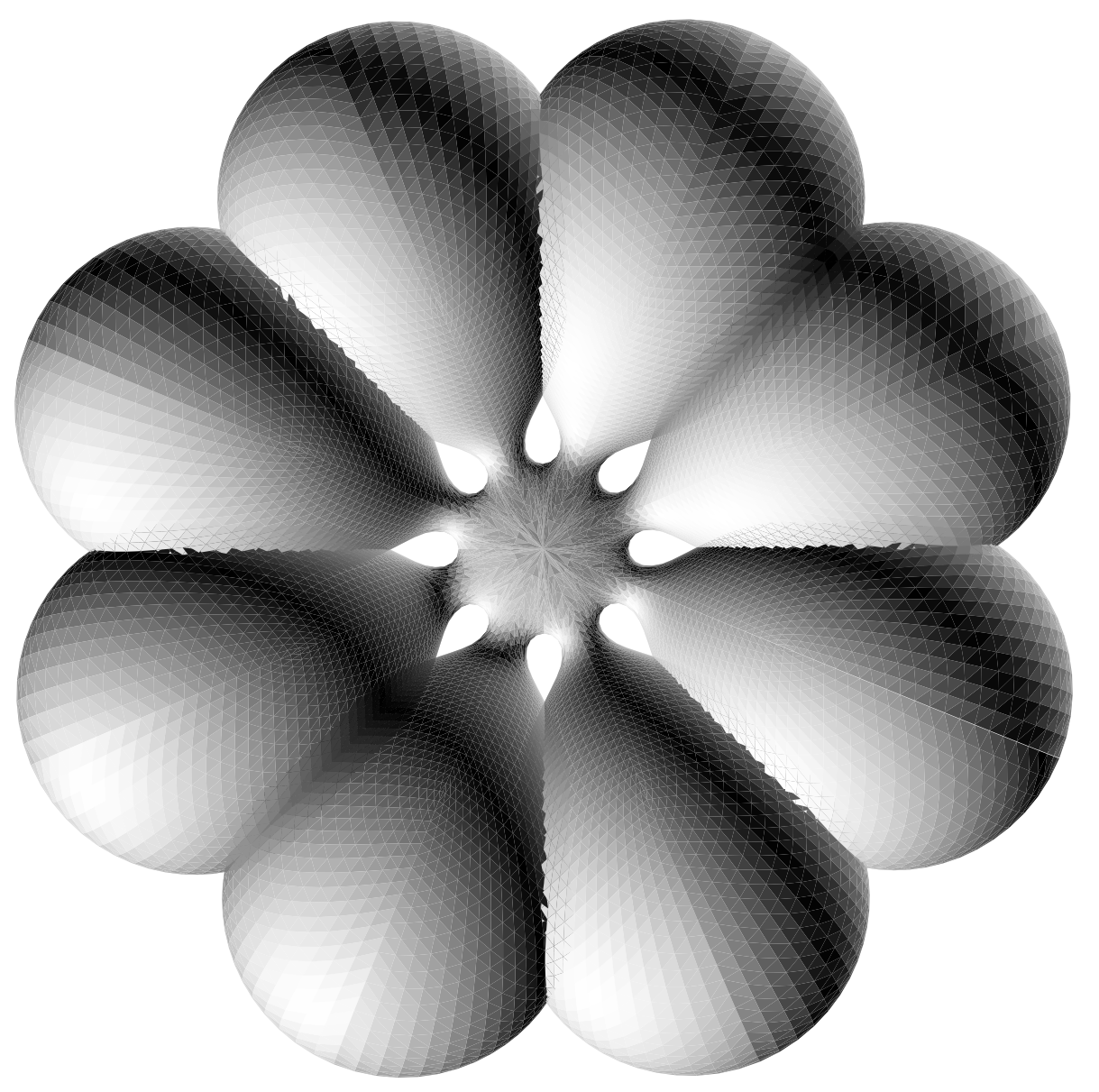

Figure 21. Noidal surface of genus 8. This surface has the same distribution of necks as the spheroidal surface, but the necks have greater force and are larger. Close to its centre the surface resembles the dihedrally symmetric 8-unduloid depicted in Figure 14.

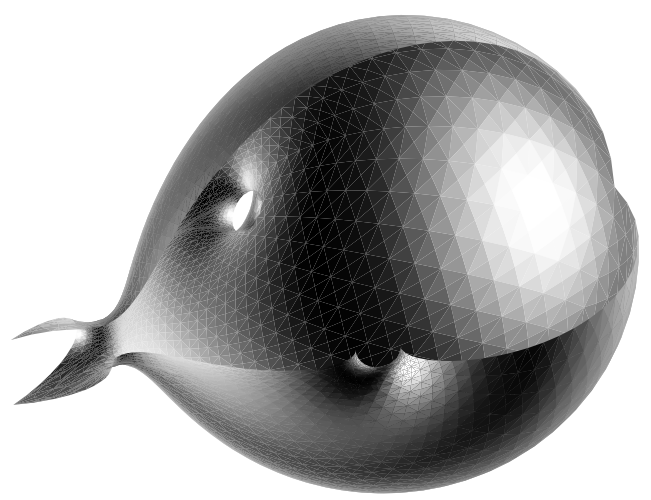

FiguRE 22. Three fundamental domains of the same surface. 


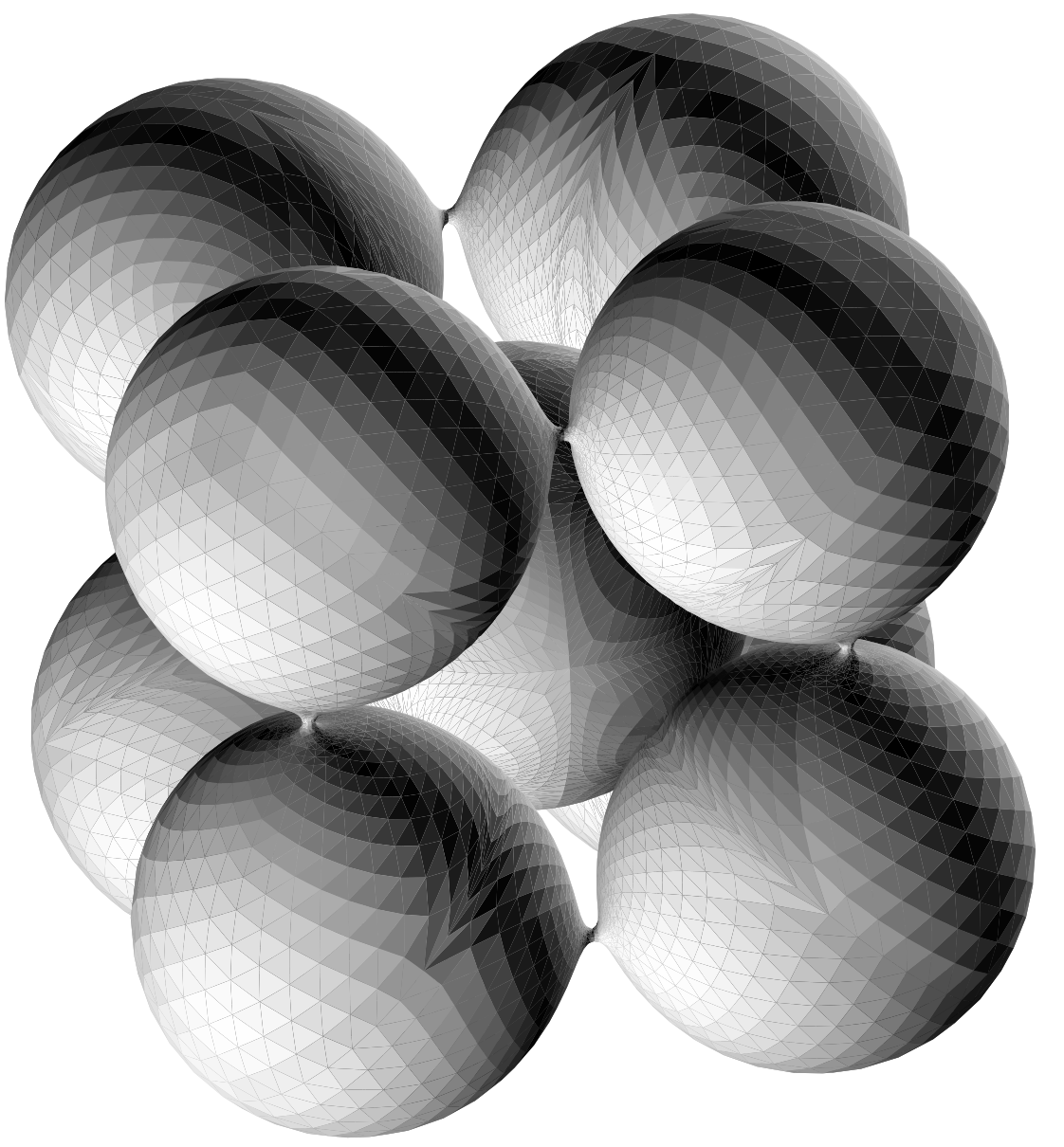

FIGURE 23. Spheroidal surface of genus 12 with cubical symmetry.

options that might soon leave the limits of our construction are to increase the number of bubbles, or to decrease symmetry.

There are many beautiful graphs with Platonic symmetry, some of which lead to further compact CMC surfaces. A description of these graphs is rather tedious, and we leave the pleasure to find them to the reader.

\section{REFERENCES}

[Alexandrov 1958] A.D. Alexandrov: "Uniqueness theorems for surfaces in the large. V", Vestnik Leningrad University, 13 (1958) no. 19, 5-8, English translation in Amer. Math. Soc. Transl. (Series 2) 21 (1962), 412-416.

[Barbosa and do Carmo 1976] J.L. Barbosa, M. do Carmo: "On the size of a stable minimal surface in $\mathbb{R}^{3} "$, Amer. J. Math. 98 (1976), 515-528.

[Bobenko 1991] A.I. Bobenko: "All constant mean curvature tori in $\mathbb{R}^{3}, \mathbb{S}^{3}, H^{3}$ in terms of theta functions", Math. Ann. 290 (1991), 209-245.

[Brakke 1992] K. Brakke: "The surface evolver", Exp. Math. 2 (1992), 141-165. 


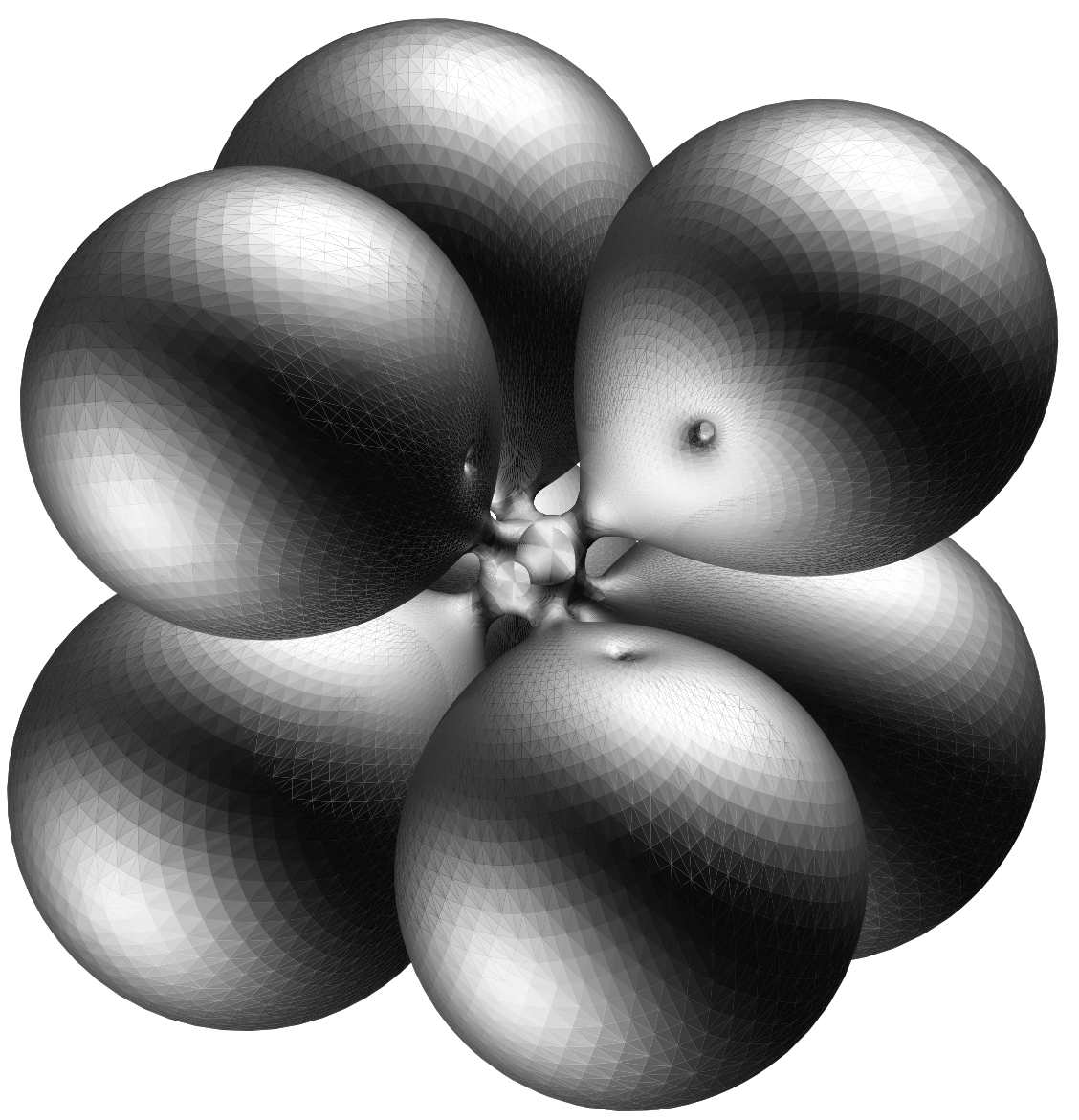

Figure 24. Noidal surface of genus 12 with cubical symmetry. One bubble is removed to give a view of the centre of the surface which resembles an 8-ended catenoid.

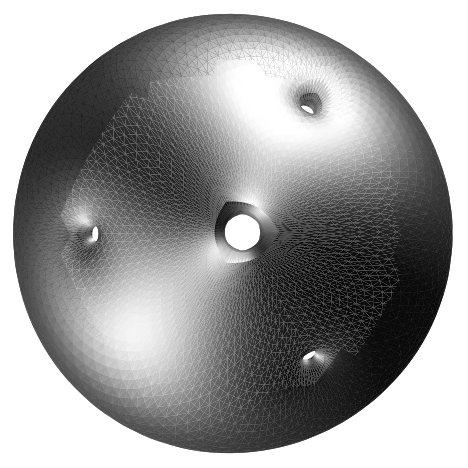

FiguRE 25. One bubble of the same surface viewed from the centre.

[Delaunay 1841] C. Delaunay: "Sur la surface de révolution, dont la courbure moyenne est constante", Journal de mathématiques 6 (1841), 309-320. 

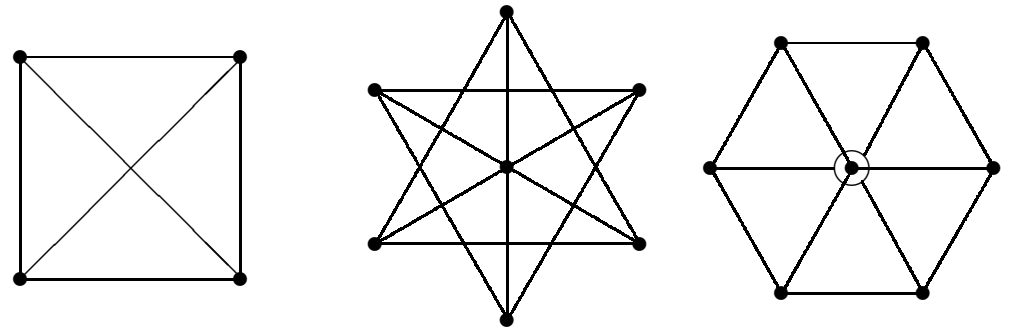

FIGURE 26. Further planar graphs with dihedral symmetry

[Ercolani et al. 1993] N. Ercolani, H. Knörrer, E. Trubowitz: "Hyperelliptic curves that generate constant mean curvature tori in $\mathbb{R}^{3}$, pp. 81-114 in Integrable systems: The Verdier Memorial Conference, Luminy 1991 (edited by O.Babelon et al.), Prog. in math. 115, Boston, Birkhäuser, 1993.

[Große-Brauckmann 1993] K. Große-Brauckmann: "New surfaces of constant mean curvature", Math. Zeit. 214 (1993), 527-565.

[Große-Brauckmann] K. Große-Brauckmann: "Constant mean curvature gyroids", GANG preprint IV-13, University of Massachusetts, Amherst 1995, and Exp. Math. submitted.

[Große-Brauckmann and Kusner] K. Große-Brauckmann, R. Kusner: "Moduli spaces of embedded constant mean curvature surfaces with few ends", in preparation

[Große-Brauckmann and Polthier 1996] K. Große-Brauckmann, K. Polthier: "Numerical examples of compact constant mean curvature surfaces", pp. 23-46 in: Elliptic and parabolic methods in geometry (B. Chow, R. Gulliver, S. Levy, J. Sullivan, eds.), A K Peters, Wellesley 1996.

[Große-Brauckmann and Polthier] K. Große-Brauckmann, K. Polthier: "Constant mean curvature surfaces derived from Delaunay's and Wente's surfaces", SFB 288 preprint, TU-Berlin (1996), also in: Visualization and Mathematics (H.-C. Hege and K. Polthier, eds.), Springer Heidelberg, 1996.

[Heil 1995] M. Heil: Numerical tools for the study of finite gap solutions of integrable systems, PhD-thesis, TU Berlin, 1995.

[Hopf 1956] H. Hopf: "Differential geometry in the large", 1956, 2nd ed., Lecture Notes in Mathematics 1000, Springer 1989.

[Karcher 1989] H. Karcher: "The triply periodic minimal surfaces of A. Schoen and their constant mean curvature companions", Man. math. 64 (1989), 291-357.

[Kapouleas 1991] N. Kapouleas: "Compact constant mean curvature surfaces in Euclidean threespace", J. Diff. Geom. 33 (1991), 683-715.

[Kapouleas 1995] N. Kapouleas: "Constant mean curvature surfaces constructed by fusing Wente tori", Invent. Math. 119 (1995), 443-518.

[Kusner 1991] R. Kusner: "Bubbles, conservation laws, and balanced diagrams", pp. 103-108 in: Geometric analysis and domputer graphics (P. Concus, R. Finn, D. Hoffman, eds.), Springer, New York, 1991.

[Korevaar et al. 1992] N. Korevaar, R. Kusner, W.H. Meeks, B. Solomon: "Constant mean curvature surfaces in hyperbolic space", Am. J. Math. 114 (1992), 1-43.

[Lawson 1970] H.B. Lawson: "Complete minimal surfaces in $\mathbb{S}^{3}$ ", Ann. of Math 92 (1970), 335-374.

[Oberknapp and Polthier] Oberknapp, B., K. Polthier: "An algorithm for discrete constant mean curvature surfaces", in: Visualization and Mathematics (H.-C. Hege and K. Polthier, eds.), Springer Heidelberg, 1996. 
[Pinkall and Polthier 1993] U. Pinkall, K. Polthier: "Computing discrete minimal surfaces and their conjugates", Exp. Math. 2 (1993), 15-36.

[Pinkall and Sterling 1989] U. Pinkall, I. Sterling: "On the classification of constant mean curvature tori", Ann. of Math. 130 (1989), 407-451.

[Wente 1986] H. Wente: "Counterexample to a conjecture of H. Hopf", Pac. J. of Math. 121 (1986), 193-243.

Universität Bonn, Mathematisches Institut, Beringstrasse 1, 53115 Bonn, GerMANY

E-mail address: kgb@@math.uni-bonn.de

Technische Universität Berlin, Fachbereich Mathematik, Ma 8-3, Strasse des 17. Juni 136, 10623 Berlin, Germany

E-mail address: polthier@@math.tu-berlin.de 